\title{
One Hundred SMUDGes in S-PLUS: Ultra-diffuse Galaxies Flourish in the Field
}

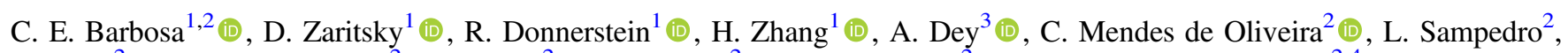 \\ A. Molino ${ }^{2}$, M. V. Costa-Duarte ${ }^{2}$, P. Coelho ${ }^{2}$ (D), A. Cortesi ${ }^{2}$ (D), F. R. Herpich ${ }^{2}$ (D), J. A. Hernandez-Jimenez ${ }^{2,4}$, T. Santos-Silva ${ }^{2}$, \\ E. Pereira $^{2}$ (D) A. Werle ${ }^{2,5}$ (D) R. A. Overzier ${ }^{2,6}$ (D) R. Cid Fernandes ${ }^{5}$ (D) A. V. Smith Castelli ${ }^{7,8}$, T. Ribeiro ${ }^{3,9}$, \\ W. Schoenell ${ }^{10,11}$ (D), and A. Kanaan ${ }^{5}$ \\ ${ }^{1}$ Steward Observatory, University of Arizona, 933 N Cherry Ave., Tucson, AZ 85719, USA; kadu.barbosa@gmail.com \\ ${ }^{2}$ Universidade de São Paulo, Instituto de Astronomia, Geofísica e Ciências Atmosféricas, Departamento de Astronomia, Rua do Matão 1225, São Paulo, SP, \\ 05508-090, Brazil \\ ${ }^{3}$ NSF's National Optical-Infrared Astronomy Research Laboratory, P.O. Box 26732, Tucson, AZ 85726, USA \\ ${ }^{4}$ Universidad Andrés Bello, Departamento de Ciencias Físicas, Fernández Concha 700, Las Condes, Santiago, Chile
${ }^{5}$ Universidade Federal de Santa Catarina, Departamento de Física, SC 88040-900, Brazil, Florianópolis, SC 88040-900, Brazil \\ ${ }^{6}$ Observatório Nacional, Ministério da Ciência, Tecnologia, Inovação e Comunicações, Rua General José Cristino, 77, São Cristóvão, 20921-400 Rio de Janeiro, RJ, \\ Brazil \\ ${ }^{7}$ Instituto de Astrofísica de La Plata, UNLP, CONICET, Paseo del Bosque s/n, B1900FWA La Plata, Argentina \\ ${ }^{8}$ Facultad de Ciencias Astronómicas y Geofísicas, UNLP, Paseo del Bosque s/n, B1900FWA, La Plata, Argentina \\ ${ }^{9}$ Universidade Federal de Sergipe, Departamento de Física, Av. Marechal Rondon, S/N, 49000-000 São Cristóvão, SE, Brazil \\ ${ }^{10}$ GMTO Corporation, 465 N. Halstead St., Suite 250, Pasadena, CA 91107, USA \\ ${ }^{11}$ Universidade Federal do Rio Grande do Sul (UFRGS), Instituto de Física, Departamento de Astronomia, Av. Bento Gonçalves 9500, Porto Alegre, RS, Brazil \\ Received 2020 January 7; revised 2020 February 7; accepted 2020 February 12; published 2020 March 16
}

\begin{abstract}
We present the first systematic study of the stellar populations of ultra-diffuse galaxies (UDGs) in the field, integrating the large area search and characterization of UDGs by the SMUDGes survey with the twelve-band optical photometry of the S-PLUS survey. Based on Bayesian modeling of the optical colors of UDGs, we determine the ages, metallicities, and stellar masses of 100 UDGs distributed in an area of $\sim 330 \mathrm{deg}^{2}$ in the Stripe 82 region. We find that the stellar masses and metallicities of field UDGs are similar to those observed in clusters and follow the trends previously defined in studies of dwarf and giant galaxies. However, field UDGs have younger luminosity-weighted ages than do UDGs in clusters. We interpret this result to mean that field UDGs have more extended star formation histories, including some that continue to form stars at low levels to the present time. Finally, we examine stellar population scaling relations that show that UDGs are, as a population, similar to other low surface brightness galaxies.
\end{abstract}

Unified Astronomy Thesaurus concepts: Low surface brightness galaxies (940); Stellar populations (1622); Stellar ages (1581); Metallicity (1031); Stellar masses (1614)

Supporting material: machine-readable table

\section{Introduction}

Ultra-diffuse galaxies (UDGs) are a recently defined class of low surface brightness (LSB) galaxy initially found in large numbers in the Coma Cluster (van Dokkum et al. 2015). Their unusually large half-light radii, $R_{e} \geqslant 1.5 \mathrm{kpc}$, for galaxies with such low central surface brightness, $\mu_{0, g} \geqslant 24$ mag $\operatorname{arcsec}^{-2}$, are striking. Although large LSB galaxies have been known for quite some time (Disney 1976; Sandage \& Binggeli 1984; Impey et al. 1988; Schombert \& Bothun 1988; Schwartzenberg et al. 1995; Dalcanton et al. 1997; Sprayberry et al. 1997), the current excitement originates from indications, either from kinematic measures of the unresolved light or globular clusters in these galaxies (Beasley et al. 2016; Toloba et al. 2018; van Dokkum et al. 2019), or from the numbers of globular clusters alone (Beasley \& Trujillo 2016; Peng \& Lim 2016; van Dokkum et al. 2017, 2018a), that at least some UDGs lie in massive $\left(>10^{11} M_{\odot}\right)$ halos.

The detection of populations of UDGs in galaxy clusters (e.g., Mihos et al. 2015; van Dokkum et al. 2015; van der Burg et al. 2016; Shi et al. 2017; Venhola et al. 2017) led to the exploration of a possible evolutionary link between these galaxies and their harsh environment (Safarzadeh \& Scannapieco 2017; Bennet et al. 2018; Conselice 2018; Ogiya 2018; Carleton et al. 2019). However, UDGs were also found in less dense environments, such as filaments (e.g., Martínez-Delgado et al. 2016), groups (e.g., Makarov et al. 2015; Smith Castelli et al. 2016; Román \& Trujillo 2017; van der Burg et al. 2017; van Dokkum et al. 2018b), the field (e.g., Leisman et al. 2017; Greco et al. 2018) and even voids (Román et al. 2019). Moreover, observational studies (e.g., Yozin \& Bekki 2015; Zaritsky 2017; Amorisco et al. 2018; Sifón et al. 2018) and theoretical ones (e.g., Amorisco \& Loeb 2016; Di Cintio et al. 2017; Rong et al. 2017; Chan et al. 2018; Jiang et al. 2019; Liao et al. 2019) found that UDGs span a wide range of physical properties and perhaps a correspondingly large range of origin stories.

A key challenge in developing a unified understanding of UDGs and their relation to other galaxies is that the data so far come from disparate studies, with different selection criteria, and mostly focus on high density environments. These deficits are exacerbated by the difficulties posed in observing such LSB galaxies. Photometric information, such as broadband colors (e.g., Prole et al. 2019), are available for many UDGs but are of limited value in determining the properties of the stellar populations, while spectroscopy, which can provide the necessary information, is only available for a small number of galaxies (e.g., Martínez-Delgado et al. 2016; Kadowaki et al. 2017; Ferré-Mateu et al. 2018; Gu et al. 2018; Ruiz-Lara et al. 2018). 
Recently, Zaritsky et al. (2019) presented the initial results from the Systematically Measuring Ultra-diffuse Galaxies (SMUDGes) survey, a systematic study to detect and characterize the photometric properties of UDGs over a large area of the sky $\left(\sim 14,000 \mathrm{deg}^{2}\right)$ using data from the Legacy survey (Dey et al. 2019). In its initial release, SMUDGes provided a catalog containing 275 UDG candidates, including most of the galaxies previously reported within $10^{\circ}$ of the Coma cluster by van Dokkum et al. (2015) and Yagi et al. (2016), using a relatively small area of the total survey $\left(334 \mathrm{deg}^{2}\right)$. SMUDGes has now analyzed the Sloan Digital Sky Survey (SDSS) Stripe 82 region and identified 172 candidate UDGs in this region, which is a much more typical region of the sky than that around the Coma cluster (D. Zaritsky et al. 2020, in preparation).

The limited passbands of the Legacy survey preclude stellar population modeling and spectroscopic observations of the SMUDGes candidates will always be highly limited (Kadowaki et al. 2017; J. Kadowaki et al. 2020, in preparation). Interestingly, the requirement of large field-of-view (FoV), multi-passband imaging in the study of UDGs intersects with the interest of several ongoing cosmological surveys, such as the JavalambrePhysics of the Accelerated Universe Astrophysical Survey (J-PAS, Benitez et al. 2014), the Javalambre Photometric Local Universe Survey (J-PLUS, Cenarro et al. 2019), and the Southern Photometric Local Universe Survey (S-PLUS, Mendes de Oliveira et al. 2019). Here we explore the synergy between SMUDGes and S-PLUS to perform the first statistical study of the stellar populations of UDG candidates over an area of sky that is not dominated by high density environments. Despite limited overlap within Stripe 82 between the two surveys, we were able to study a sample of 100 UDG candidates and perform the largest detailed population study of these galaxies to date.

This paper is structured as follows. In Section 2, we describe the two data sets used in this work. In Section 3, we describe the photometry of the UDG candidates in the context of S-PLUS, and in Section 4, we present the method developed to determine their stellar populations using a Bayesian framework. In Section 5, we present our results and discuss the main implications of our work for our understanding of the nature of UDGs. We conclude and summarize this work in Section 6. Throughout, we assume a standard $\Lambda \mathrm{CDM}$ cosmology whenever necessary, assuming $H_{0}=70 \mathrm{~km} \mathrm{~s}^{-1} \mathrm{Mpc}^{-1}$. All magnitudes use the $\mathrm{AB}$ system (Oke 1964; Oke \& Gunn 1983).

\section{Data}

\subsection{SMUDGes Sample}

Our ability to locate LSB galaxies has been limited both by the lack of sensitivity and instrumental constraints, and various attempts have been made to optimize observations at low surface brightness (e.g., Gonzalez et al. 2001; Abraham \& van Dokkum 2014; Mihos et al. 2015). However, there have been no systematic attempts to use current, large volume archival data to search for LSB galaxies, which have not been identified before because standard pipelines are not optimized find such systems. The SMUDGes project (Zaritsky et al. 2019) was conceived to develop an automated way to search for LSB galaxies over a large area of the sky using data from the Legacy imaging survey (Dey et al. 2019), a deep three-band observational campaign that supports the Dark Energy Spectroscopic Instrument (DESI) project (Schlegel et al. 2011; DESI Collaboration et al. 2016a, 2016b).
Most of what is known about UDGs as a population is based on observations of the Coma cluster (van Dokkum et al. 2015; Yagi et al. 2016). In this initial stage of the project, the SMUDGes detection algorithm has been constrained to search UDGs similar to those found in Coma, and thus is focused on systems with angular sizes $R_{e} \gtrsim 5^{\prime \prime}$, which are easier to classify than smaller objects in the absence of redshift information. The methodology used to identify UDGs is described in detail in Zaritsky et al. (2019) and D. Zaritsky et al. (2020, in preparation), and here we summarize the main steps of the process. First, bright, saturated sources are detected, modeled, and replaced in the DESI images by background noise, whereas fainter background and foreground sources are carefully modeled and subtracted. Then, wavelet filtering is used to detect sources according to size and surface brightness criteria, defined to have $\mu_{0, g} \geqslant 24 \mathrm{mag} \operatorname{arcsec}^{-2}$ and $R_{e}>5$ !' 3 . Finally, all UDG candidates are modeled with a single Sérsic component using GALFIT (Peng et al. 2002, 2010).

In this work, we use a sample of 172 UDG candidates in the Stripe 82 area. From SMUDGes we adopt the values of $m_{g}, m_{r}, m_{z}$, Sérsic index $n$, and $R_{e}$ in arcseconds, position angle, and axis ratio. Without distance estimates, we cannot determine whether these systems pass the common defining criteria for UDGs, $R_{e} \geqslant 1.5 \mathrm{kpc}$, and some of these galaxies may actually be dwarf galaxies at small distances. The redshift by association (defining high density regions in terms of normal galaxies and assigning SMUDGes to the redshift of that overdensity) worked for 25 candidates in Stripe 82 and all 25 satisfy the $R_{e}>1.5 \mathrm{kpc}$ criterion at the assigned distance. Only one has $R_{e}>6 \mathrm{kpc}$, which seems to be about the upper limit on size-it has $R_{e}=8.6 \mathrm{kpc}$ - which suggests that this one may have the wrong redshift. In this particular case, the UDG candidate is close in projection to a nearby bright galaxy and so it may instead be a satellite of that galaxy (D. Zaritsky et al. 2020 , in preparation). Therefore, we work under the hypothesis that we have a sample of UDGs with low contamination by dwarf galaxies, but we examine this issue again further below.

\subsection{S-PLUS DR1 Data}

We use data from the S-PLUS first data release (DR1), which covers an area of $336 \mathrm{deg}^{2}$ in the Stripe 82 equatorial field, observed with the $\mathrm{T} 80 \mathrm{~S}$, a $0.8 \mathrm{~m}$ robotic telescope with a wide FoV of $\sim 1.8 \mathrm{deg}^{2}$, located in Cerro Tololo, Chile. The DR1 data are already reduced and are publicly available in the NSF's National Optical-Infrared Astronomy Research Laboratory archive. ${ }^{12}$ Details about the survey strategy and data reduction process are described by Mendes de Oliveira et al. (2019), while the photometric calibration is described in L. Sampedro et al. (2020, in preparation).

The main survey strategy is aimed at obtaining large coverage of the southern sky $\left(\sim 9000 \mathrm{deg}^{2}\right)$ for astronomical and cosmological studies in the local universe. The S-PLUS uses the same photometric system as the J-PLUS survey (Marín-Franch et al. 2012), which consists of twelve optical bands, including 5 broad bands similar to those used by the SDSS ugriz system, and a set of seven narrow-band $(\Delta \lambda=100-200 \AA)$ filters placed at various rest-frame optical features, including [O II] $\left(\lambda_{\text {eff }}=3771 \AA\right.$ A), Ca H $+\mathrm{K}\left(\lambda_{\text {eff }}=3941 \AA\right), \mathrm{H} \delta\left(\lambda_{\text {eff }}=4094 \AA\right)$, G-band $\left(\lambda_{\text {eff }}=4292\right.$ $\AA$ ), $\mathrm{Mg} b$ triplet $\left(\lambda_{\text {eff }}=5133 \AA\right), \mathrm{H} \alpha\left(\lambda_{\text {eff }}=6614 \AA\right.$ ) , and the $\mathrm{Ca}$ triplet $\left(\lambda_{\text {eff }}=8611 \AA\right.$ ). Considering a signal-to-noise ratio $(\mathrm{S} / \mathrm{N})$

\footnotetext{
12 https://datalab.noao.edu/splus/index.php
} 


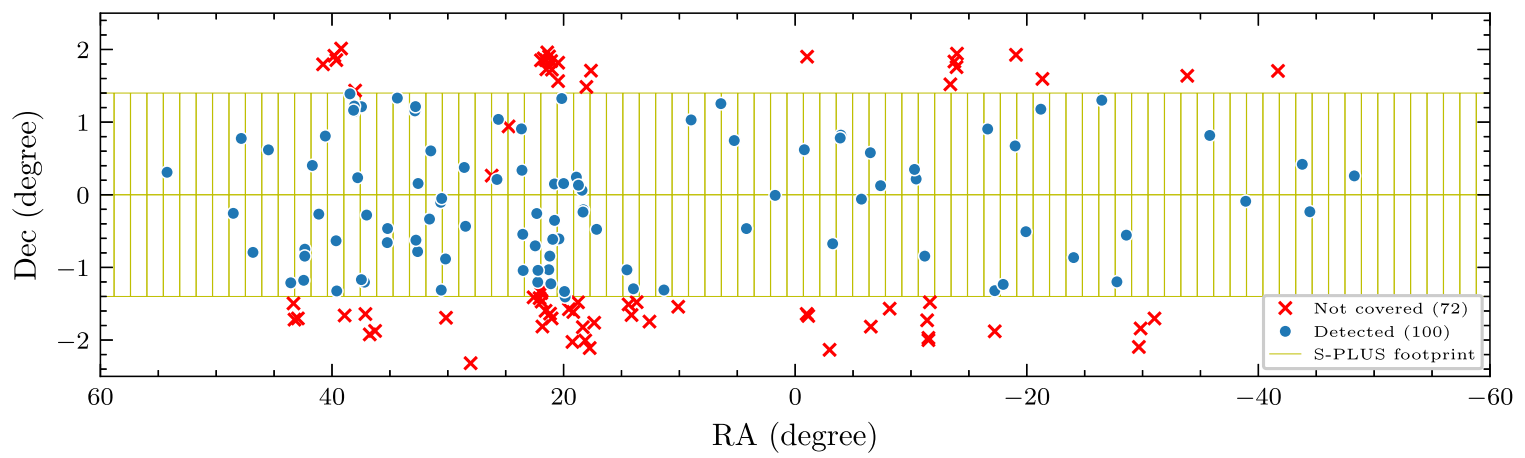

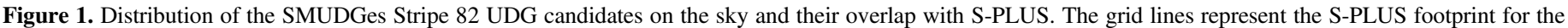

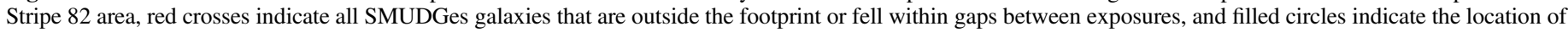
all UDG candidates with S-PLUS data.

threshold of 3 , the survey is complete in the broad bands to magnitudes of $u=21.07, g=21.79, r=21.6, i=21.22$, and $z=20.64$, whereas it is complete to magnitudes of $\sim 20.4$ in all narrow bands (Mendes de Oliveira et al. 2019).

The S-PLUS DR1 data cover Stripe 82 using a pair of exposures at each R.A., limiting the decl. to the range $-1.4 \leqslant$ decl. $\leqslant+1.4$. Moreover, the S-PLUS DR1 data did not use dithering, causing occasional gaps between exposures, resulting in a few UDGs that are not observed despite being within the footprint of the survey. In total, we have observations for only 100 SMUDGes from the initial sample of 172 . Figure 1 shows the spatial distribution of the SMUDGes Stripe 82 sample overlapped with the S-PLUS DR1 footprint.

\section{Photometry of UDGs from the S-PLUS Data}

UDGs are not easily detected given the surface brightness limits of the S-PLUS survey, and only one UDG was previously detected in the DR1 catalog of photometric redshifts (Molino et al. 2019). Therefore, we had to obtain our own photometry of the UDGs from the S-PLUS images leveraging the information from the deeper SMUDGes photometry.

Regarding the data quality of S-PLUS, all images in the S-PLUS Main Survey, which includes Stripe 82, were obtained during photometric nights with seeing $\leqslant 2^{\prime \prime}$. Among the 61 different tiles used in this work, the mean full width at half maximum (FWHM) over all bands is 1".4. Moreover, because each field is imaged in all bands consecutively in a given observational block, there are only small seeing variations among all bands for each tile (mean standard deviation among bands of 0 .'14). We conclude that there is no need to homogenize the seeing across the images for our photometry.

For each UDG, we perform aperture photometry in each of the 12 bands from S-PLUS using the PHOTUTILS package (Bradley et al. 2019). To ensure consistent photometry, for each UDG we define an elliptical aperture with a semimajor axis length of $R_{e}$, and location, position angle, and ellipticity determined from the GALFIT Sérsic profile fitting from the SMUDGes analysis. We subtract local sky using an elliptical annulus with inner and outer radii of $2.5 R_{e}$ and $4 \mathrm{R}_{\mathrm{e}}$, respectively. Presuming that the Sérsic profile is a good approximation to the surface brightness profile of the UDGs, this annulus is large enough to avoid contamination of the sky region by the galaxy itself (see Graham \& Driver 2005). We use sigma clipping to remove the contribution of other sources when we estimate the median background. All observed magnitudes are corrected for the foreground Galactic extinction using the dust maps from Schlegel et al. (1998) recalibrated by Schlafly \& Finkbeiner (2011), assuming that $R_{V}=3.1$ for the Milky Way (Savage \& Mathis 1979).

The aperture photometry method described above has the advantage of allowing the detection of most UDGs in individual bands despite their low $\mathrm{S} / \mathrm{N}$. However, in most cases (87 galaxies), at least one band had a nondetection, as the measured flux inside the galaxy is smaller than the flux in the sky annulus. In these cases, we are only able to set an upper limit on the source flux. There are missing detections in most of the bands, but the blue bands are the most affected, in particular the narrow bands F378 and F395, for which there are flux detections in only $\sim 60 \%$ of the galaxies. Nevertheless, in the majority of cases (97 galaxies), we have flux detections in at least six bands, which already provides better spectral coverage in the optical than do the SDSS bands, and $80 \%$ of the galaxies have detected flux in at least nine bands.

In Figure 2, we show a sample of detection images of SMUDGes UDGs, produced by stacking all 12 S-PLUS bands, ordered in decreasing central surface brightness in the $g$ band, $\mu_{0, g}$, and highlight the photometric apertures.

\section{Stellar Populations from Multiband Observations}

We quantify the properties of the stellar populations of our sample by performing spectral energy distribution (SED) fitting of the galaxies in all detected bands of the S-PLUS data. Considering that the star formation history (SFH) of galaxies is difficult to determine from photometric data alone, and that simulations indicate that UDGs may have bursty SFHs (Di Cintio et al. 2017; Chan et al. 2018), we assume that SEDs may be described by a single stellar population (SSP), such that

$$
f_{\lambda}(\lambda)=f_{0} \cdot \operatorname{SSP}([\mathrm{Fe} / \mathrm{H}], \text { Age }, z) 10^{-0.4 A_{\lambda}},
$$

where $f_{0}$ is a scale factor for the spectral flux density of the galaxy, SSP represents a single stellar population model that depends on the metallicity $([\mathrm{Fe} / \mathrm{H}])$, age, and the redshift $z$ of the galaxy, and $A_{\lambda}$ represents a dust-screen attenuation model. One important cautionary point about the use of SSPs to represent a potentially more complicated $\mathrm{SFH}$ is that the derived properties are luminosity weighted. As appreciated previously (see Serra \& Trager 2007), luminosity-weighted ages are expected to be biased toward the youngest populations, in contrast to the luminosity-weighted metallicity, which reflects more closely the mass-weighted average. 

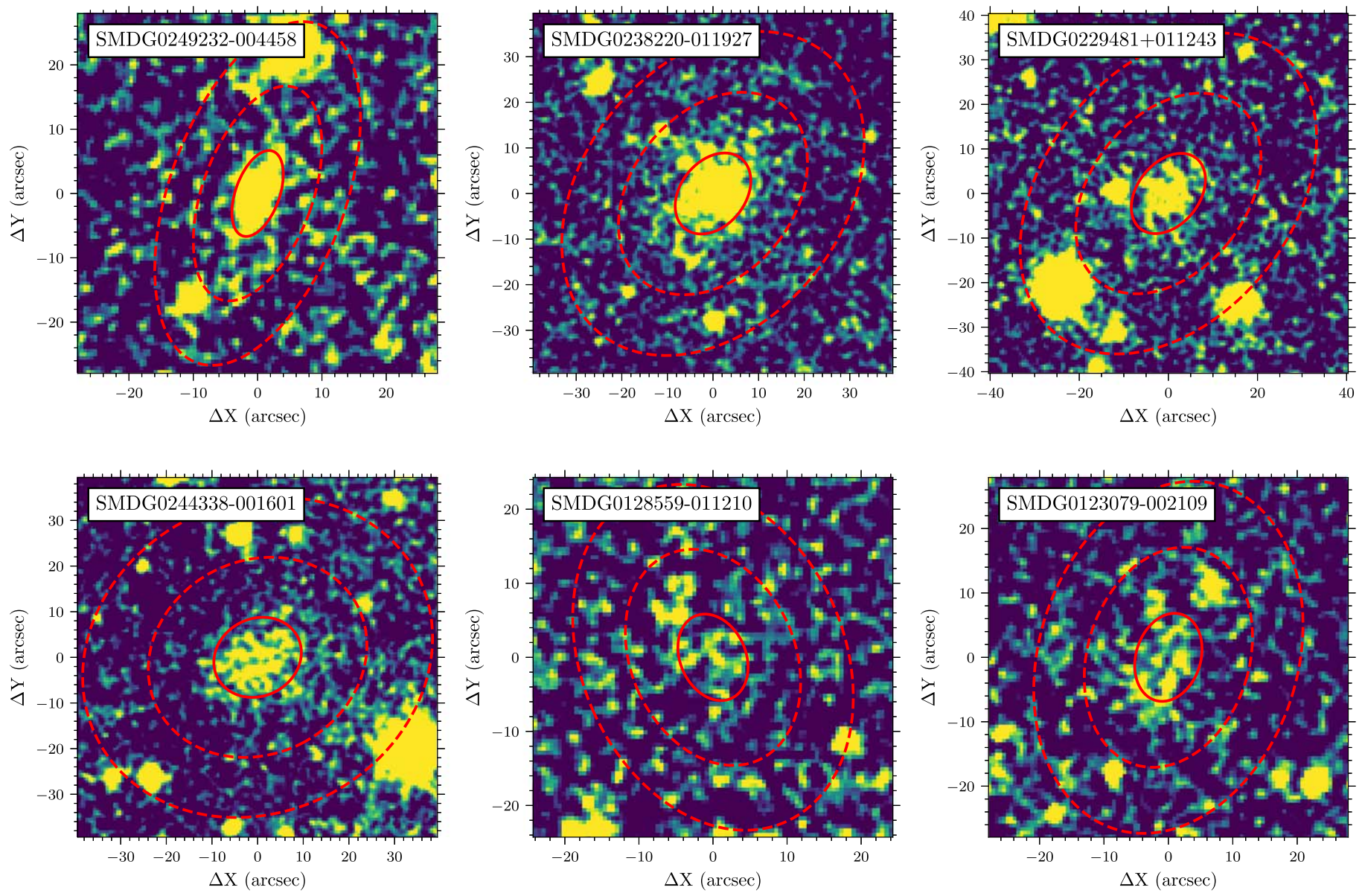

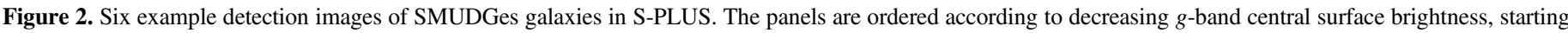
in the upper left. The solid red ellipses outline the $1 R_{\mathrm{e}}$ photometric aperture while the dashed red lines indicate the annulus used for background estimation.

Considering both the low $\mathrm{S} / \mathrm{N}$ of the observations and the low spectral resolution of the photometric system, we expect that derived parameters may be correlated, as is the case in the wellknown age-metallicity degeneracy problem (Worthey 1994), and that some parameters will not be properly estimated. Therefore, we use a Bayesian statistical model to fit the SED of the galaxies and to estimate the stellar population parameters. Using this approach, we can identify possible parameter correlations and provide uncertainties that are marginalized over the distribution of all the other parameters in the model.

Bayes' theorem allows for the inference of the probability distribution of a set of parameters $\theta$ in a statistical model based on a data set $D$ using the relation

$$
p(\theta \mid D) \propto p(\theta) p(D \mid \theta),
$$

where $p(\theta \mid D)$ represents the posterior probability distribution of the parameters $\theta$ given the data $D, p(\theta)$ represents the prior distribution of the parameters, and $p(D \mid \theta)$ is the likelihood distribution (see, e.g., Gelman et al. 2004). Below we describe the priors for all of the parameters in our model.

\subsection{Prior and Likelihood Distributions}

The flux scale factor $f_{0}$ can vary by orders of magnitude depending on the brightness of the source. Therefore, it is more convenient to model its logarithm, which can be described by the prior

$$
\log f_{0} \sim \operatorname{Normal}\left(\mu_{0}, \sigma_{0}^{2}\right),
$$

where $\mu_{0}$ and $\sigma_{0}^{2}$ indicate the mean and the variance of the distribution, respectively. In practice, we estimate $\mu_{0}$ using the data, and we assume $\sigma_{0}=3$ to allow a large range of magnitudes.

Our modeling is parameterized in terms of two stellar population parameters, the age and metallicity, whose priors are set by the limits of the model ranges. In this work, we use the E-MILES models (Vazdekis et al. 2016), assuming prior distributions given by

$$
[\mathrm{Fe} / \mathrm{H}](\mathrm{dex}) \sim \operatorname{Uniform}(-1.79,0.4)
$$

and

$$
\text { Age }(\text { Gyr }) \sim \operatorname{Uniform}(0.4,14) .
$$

The main reason to set the limits above is to ensure that the SSP models have good quality in the ultraviolet according to the classification of Vazdekis et al. (2010), resulting in the exclusion of SSP models with $[\mathrm{Fe} / \mathrm{H}]=-2.27$, which may not be appropriate for metallicity estimation. Additionally, we also require a regular grid in the parameter space to perform linear interpolation of the SSP models, allowing a continuous coverage of ages and metallicities. As a consequence, we had to restrict the models to ages greater than $0.4 \mathrm{Gyr}$ because part of the young SSP models are not extended to the near-infrared, 
which is necessary to cover the S-PLUS $z$ band properly. In particular, we adopt SSP models with a bimodal initial mass function (IMF), a piecewise function defined by Vazdekis et al. (1996) that matches the Salpeter IMF for masses $>0.8 M_{\odot}$ but is flattened at lower masses similarly to the Milky Way IMF (e.g., Chabrier 2003). Given that the current version of the E-MILES stellar population models does not include the abundance of individual or alpha elements yet, we are restricted to the base models, which assume that $[\mathrm{M} / \mathrm{H}]=[\mathrm{Fe} / \mathrm{H}]$ at solar metallicity. However, this assumption does not hold at low metallicities because the Milky Way stars used in the computation of the models are themselves alpha enhanced (see Vazdekis et al. 2010). The consequences of possible offsets resulting from nonsolar abundance ratios are discussed further below.

The redshifts of our galaxies are of great interest because they set the distances to the galaxies and their physical parameters, and allow a proper classification of the candidates as UDGs. Without additional spectroscopic redshift or redshift by association for our sample, we consider a prior that takes into consideration a few assumptions. LSB galaxies with angular sizes $R_{e} \gtrsim 5^{\prime \prime}$ have only been associated to environments with distances smaller than $100 \mathrm{Mpc}$ (see González et al. 2018), thus we can assume all UDG candidates are nearby. Moreover, all UDG candidates were selected with a minimum effective radius of $R_{e}=5$ !" 3, which implies a physical radius of $R_{e}=2.5 \mathrm{kpc}$ at the distance of Coma, $100 \mathrm{Mpc}$, or a redshift of $z \approx 0.023$. At a distance as low as $200 \mathrm{Mpc}$, or $z \approx 0.046$, these UDGs would all already have an effective radius of $R_{e} \geqslant 5 \mathrm{kpc}$, which is larger than most UDGs found so far (e.g., Venhola et al. 2017). We conclude that it is very unlikely that many of our candidates lie at $z>0.04$. Based on these considerations, we use the prior

$$
z \sim \operatorname{HalfNormal}\left(0.03^{2}\right),
$$

where we adopt the half-normal distribution to restrict the redshifts to positive values, and we assume a variance of $0.03^{2}$. In practice, this prior implies a median redshift $z \approx 0.02$, similar to Coma, with peak probability at $z=0$.

Regarding the dust attenuation, our data include only wavelengths $\lambda>3000 \AA$ for low redshift galaxies, avoiding the $2175 \AA$ bump (Stecher 1965). For these wavelengths, most of the extinction laws, such as those obtained for the Milky Way (Allen 1976; Fitzpatrick \& Massa 1986), the Large Magellanic Cloud (Fitzpatrick \& Massa 1986), the Small Magellanic Cloud (Prevot et al. 1984; Bouchet et al. 1985), and starburst galaxies (Calzetti et al. 2000), agree (see Werle et al. 2019). We adopt a parameterized extinction law from Cardelli et al. (1989), which depends on two parameters, the total extinction in the $V$-band, $A_{V}$, and the total-to-selective extinction, $R_{V}$. The total extinction is modeled according to the prior

$$
A_{V} \sim \operatorname{Exponential(0.2)}
$$

where 0.2 represents the mean value of the exponential distribution. This prior restricts the value of the extinction to positive values and also favors smaller extinction values rather than large. We also allow $R_{V}$ to vary in our models using the prior

$$
R_{V} \sim \operatorname{Normal}^{+}(3.1,1 .),
$$

which assumes that the total-to-selective extinction is similar to that measured generally within the Milky Way
(Savage \& Mathis 1979). The plus signal indicates that we restrict $R_{V}$ to positive values.

Finally, it is necessary to define a log-likelihood for the use of the Bayes' theorem. The widely common assumption is that the observed SED consists of independent, normal random variables, and thus the log-likelihood can be simply described as a $\chi^{2}$ distribution. However, the accuracy of the model determined using the normal assumption may be compromised if the observations contain outliers (see Vanhatalo et al. 2009). In observational settings, the causes of outliers may be either external to the source, such as contamination by cosmic rays or the incomplete removal of sky, or internal to the source, as is the case when the model is incomplete, for example when it does not account for emission lines.

Emission lines have been directly observed in optical observations of at least one cluster UDG (Kadowaki et al. 2017), and may be common in field UDGs (Leisman et al. 2017). Observationally, emission lines systematically inflate the observed fluxes in passbands in which they appear, an effect that is likely to be most noticeable in the bluer, narrow bands. However, the modeling of emission lines requires the inclusion of secondary stellar populations with young ages $(<0.01 \mathrm{Gyr})$ and/or post-asymptotic giant branch stars (Byler et al. 2017) plus a prescription for nebular emission (e.g., Fioc \& Rocca-Volmerange 1999; Leitherer et al. 1999). To simplify the modeling, we instead adopt a robust fitting approach that may deal with outliers, including possible emission lines, adopting a Student's $t$-distribution log-likelihood.

Similar to the normal distribution, the Student's $t$-distribution is a symmetric and bell-shaped distribution, but with long tails that allow for a nonnegligible probability far from the center of the distribution (see Gelman et al. 2004). Assuming that we are modeling $N$ discrete bands in a given SED, the loglikelihood that we map is given by

$$
\begin{aligned}
\ln p(D \mid \theta)= & N \log \left[\frac{\Gamma\left(\frac{\nu+1}{2}\right)}{\sqrt{\pi(\nu-2)} \Gamma\left(\frac{\nu}{2}\right)}\right] \\
& -\frac{1}{2} \sum_{i=1}^{N} \log \sigma_{i}^{2} \\
& -\frac{\nu+1}{2} \sum_{i=1}^{N} \log \left[1+\frac{\mu_{i}^{2}}{\sigma_{i}^{2}(\nu-2)}\right],
\end{aligned}
$$

where $\Gamma(x)$ represents the gamma function of variable $x, \nu$ represents the degrees of freedom of the Student's $t$-distribution, $\sigma_{i}$ represents the uncertainties of a given SED for the $i$ th band, and the mean $\mu_{i}$ represents the difference between the observed and the model SED. The Student's $t$-distribution log-likelihood does not depend solely on the data and its uncertainties, but also on the value of another variable, $\nu$, which controls the amount of weight on the tails of the Student's $t$-distribution. For instance, if $\nu \rightarrow 2$, the tails of the distribution have more weight in the distribution, whereas if $\nu \rightarrow+\infty$, the distribution tends to a normal distribution. We also model the value of $\nu$ during the $\log$ likelihood mapping assuming a noninformative prior for the degrees of freedom given by

$$
\nu \sim \operatorname{Uniform}(2,50),
$$

which is required to be open ended only in the lower bounds to avoid the undefined likelihood that occurs if $\nu=2$. 


\subsection{Sampling and Results}

To deploy the above SED fitting modeling in the context of the S-PLUS project, we developed a Bayesian SED fitter (BSF; C. E. Barbosa 2020, in preparation) as a general tool to model either SEDs or spectra of galaxies. BSF is written in the Python programming language based on the PYMC3 statistical package (Salvatier et al. 2016), which allows for the construction of general Bayesian models while abstracting the complex issues related to the actual modeling and sampling. An attractive feature of the PYMC3 package, not found in other commonly adopted packages such as the EMCEE (Foreman-Mackey et al. 2013), is the implementation of the No U-Turn Sampler (NUTS, Hoffman \& Gelman 2011), a Hamiltonian Monte Carlo sampler that has been shown to perform well in complex, multidimensional problems without the need of manual tuning. This sampler works better than other traditional samplers, such as the Metropolis-Hastings algorithm (Hastings 1970) and the Gibbs sampler (Gelfand \& Smith 1990), in problems with highly correlated variables in a space parameter with hundreds of dimensions (see Hoffman \& Gelman 2011).

As discussed in Section 3, there are a number of nondetections in our photometry. To simplify our modeling, we only included detected bands in the SED fitting for each UDG, and we leave the modeling including nondetections for forthcoming work. The samples from the posterior distributions were generated with BSF in four chains with 500 burn-in interactions and 500 draws. Figure 3 shows the comparison between the observations and the models for the sample of galaxies previously shown in Figure 2.

To illustrate the process of obtaining representative values and uncertainties for the model parameters, Figure 4 shows the posterior samples determined with BSF for two of the UDGs presented in Figure 2: SMDG0123079-002109, representing a relatively faint galaxy and SMDG0238220-011927, representing a relatively bright galaxy. Throughout our analysis, we use the median to determine the representative value of all parameters, and we use the percentile values of $16 \%$ and $84 \%$ to estimate the $1 \sigma$ confidence intervals of the parameters, always using the marginalized posterior distribution, shown in the histograms. In Table 1, we present the results of this analysis for the first ten entries of the SMUDGes sample. The full table is available online in machine-readable format.

\subsection{Stellar Masses}

We determine the stellar mass of each UDG combining our SED fitting results of the S-PLUS data with the photometric properties measured in the deeper SMUDGes images. We adopt two different approaches. First, we use the SED fitting photometric redshift to estimate the distance and the total apparent $r$-band magnitude from SMUDGes to determine the total magnitude, assuming a Hubble-Lemaitre law with $H_{0}=70 \pm 2 \mathrm{~km} \mathrm{~s}^{-1} \mathrm{Mpc}^{-1}$. Next, we use the $r$-band massto-light ratio from the E-MILES models (Vazdekis et al. 2010; Ricciardelli et al. 2012) to obtain the total stellar mass, assuming that $M_{\odot, r}=4.65$ (Willmer 2018). These calculations are performed using the Monte Carlo chains, and thus the uncertainties are marginalized over all parameters in the SED fitting model. Second, we estimate the stellar mass using the color-mass relation from Taylor et al. (2011), which is based on data from the Galaxy And Mass Assembly survey (Driver et al. 2009, 2011). Reassuringly, the stellar masses resulting from the two approaches always agree to within $0.1 \mathrm{dex}$, which is a difference that is much smaller than the typical mass uncertainties $(\sim 0.8$ dex $)$. We conclude that our stellar mass estimates are likely to be dominated by internal random uncertainties rather than by a systematic error in the conversion between luminosity and stellar mass. For the remainder of this work, we use the stellar masses determined using the first method. The stellar masses derived by the first method are also presented in Table 1.

\subsection{Evaluating the Posterior Distributions}

To understand how well we constrain the parameters in our model, we compare the posterior distributions with the prior distribution. We perform this exercise in Figure 5, where we show the posterior medians and uncertainties for five free parameters in our model $\left(A_{V}, R_{V}, \operatorname{Age},[\mathrm{Fe} / \mathrm{H}]\right.$, and $\left.z\right)$ as a function of $m_{r}$, the apparent magnitude of the galaxies according to the SMUDGes measurements. Overall, the fitting is better constrained, i.e., is restricted to a narrower range of values in the posterior distribution, for the brighter sources $\left(m_{r} \lesssim 19\right)$, while the posterior distributions tend to be more similar to the prior distributions for the fainter sources $\left(m_{r} \gtrsim 19\right)$. We discuss the results for the individual parameters below.

The extinction law parameters have limited impact on the optical SED shape of the UDGs, and no strong dust attenuation was required to fit the models. The median total extinction of $A_{V} \approx 0.1-0.2$ is recovered in all cases, whereas the total-toselective extinction $R_{V}$ is mostly unchanged from the prior distribution. In practice, both parameters have the role of nuisance parameters in our analysis, as they are not of direct interest for this work, but are still taken into consideration in the analysis of the stellar populations parameters and the redshift.

The metallicity clearly departs from the prior distribution in most cases, with median metallicities systematically small $([\mathrm{Fe} / \mathrm{H}] \approx-1 \mathrm{dex})$. Even though the $1 \sigma$ uncertainties remain similar to the prior for the faint UDGs, the posterior distributions for the metallicity are usually skewed toward low metallicities in most cases, and are not flat shaped like the priors. The main concern in the derived metallicities occurs for the more metal-poor galaxies, given that they are sometimes compatible with the lowest metallicity available in our SSP grid $([\mathrm{Fe} / \mathrm{H}]=-1.79)$. Without a larger grid of models, we cannot rule out that some of these systems have even lower metallicities. Overall, however, we conclude that our metallicity estimates are well constrained by our SED fitting.

Similarly, despite the large uncertainties for the faint UDGs, we do find that the luminosity-weighted ages tend to be smaller than the prior median (age $\approx 7 \mathrm{Gyr}$ ). One important point in the evaluation of the ages is that we can see more variation in the SED's of younger galaxies, in the sense that it is easier to differentiate between a 1 and a $2 \mathrm{Gyr}$ old population than to differentiate between a 10 and a 12 Gyr old population. We see that effect in practice in our modeling in Figure 5, as the posterior distributions for galaxies with young ages are usually narrower than the prior distribution, while for those with old ages we tend to obtain relatively flat posteriors. Overall, we conclude that we are able to differentiate between young 

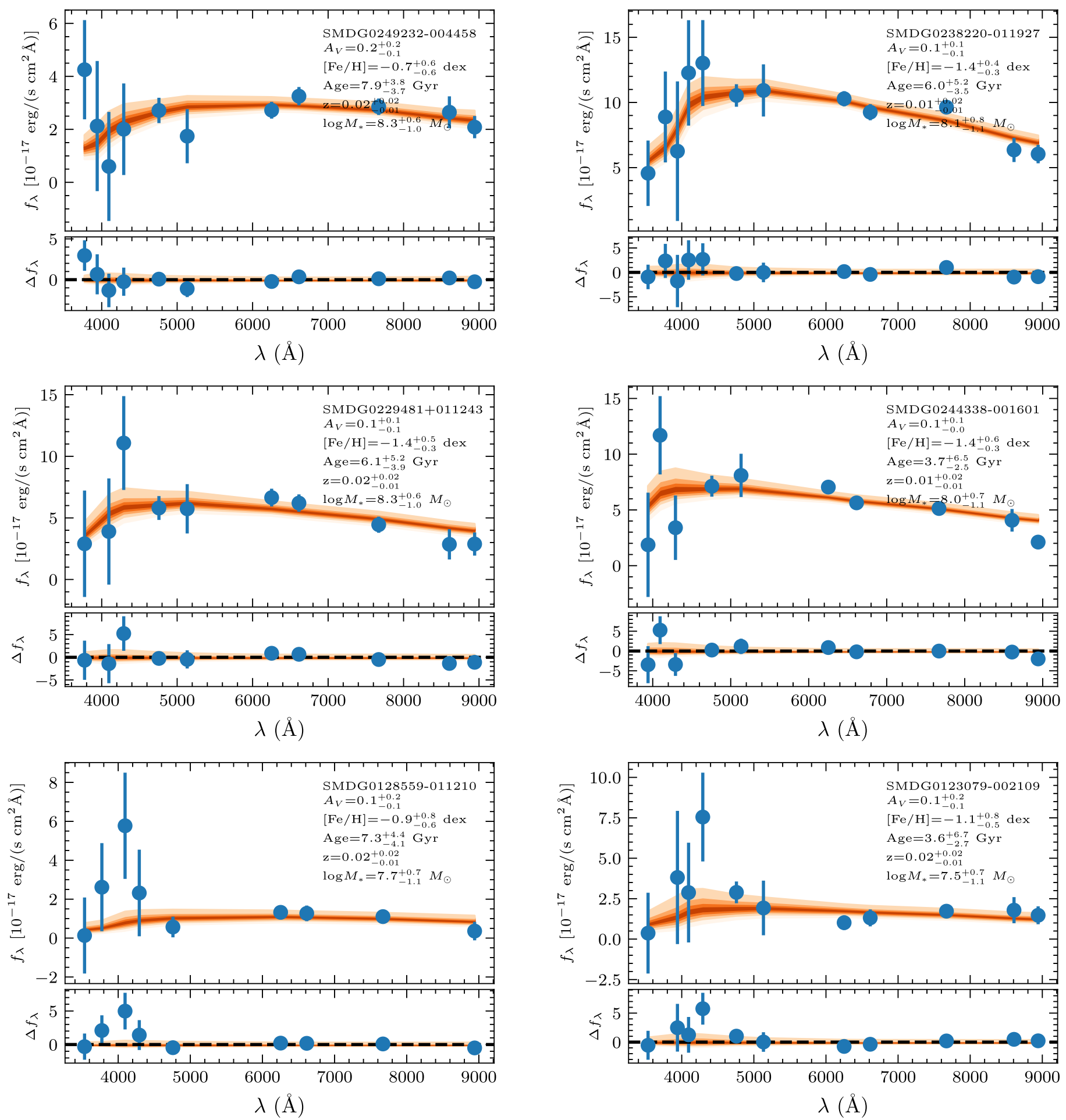

Figure 3. Resulting SED fits for the six examples presented in Figure 2. The blue filled circles represent the S-PLUS flux densities in detected bands. The shaded regions indicate the confidence percentile levels of the SED fitting, from 5\% to $95 \%$ in intervals of $10 \%$. Each panel comes in two portions, where the upper area shows the data and SED fit and the lower shows the residuals. A summary of the most relevant parameters is included in upper right of each panel.

and old stellar populations in our UDG candidates, which is enough to allow a broad discussion of the formation of these systems.

Finally, the quality of the modeled photometric redshifts also depends on the apparent magnitude of the UDGs. The posterior redshift distribution for the faint UDGs is very similar to the prior distribution. In these cases, the quoted uncertainties in the redshift are around $0.8 \mathrm{dex}$, which is simply the propagation of the allowed prior range. On the other hand, the bright UDGs have a narrower range of redshifts in the posterior distribution, and their median redshifts are usually smaller than the prior median of $\approx 0.02$. However, even in these cases, the redshift estimate is very uncertain, and we are only able to constrain the photometric redshift with errors $\sigma_{z} \approx 0.01$ in the best cases. This has important implications in the classification of the UDG candidates, as we discuss below. 

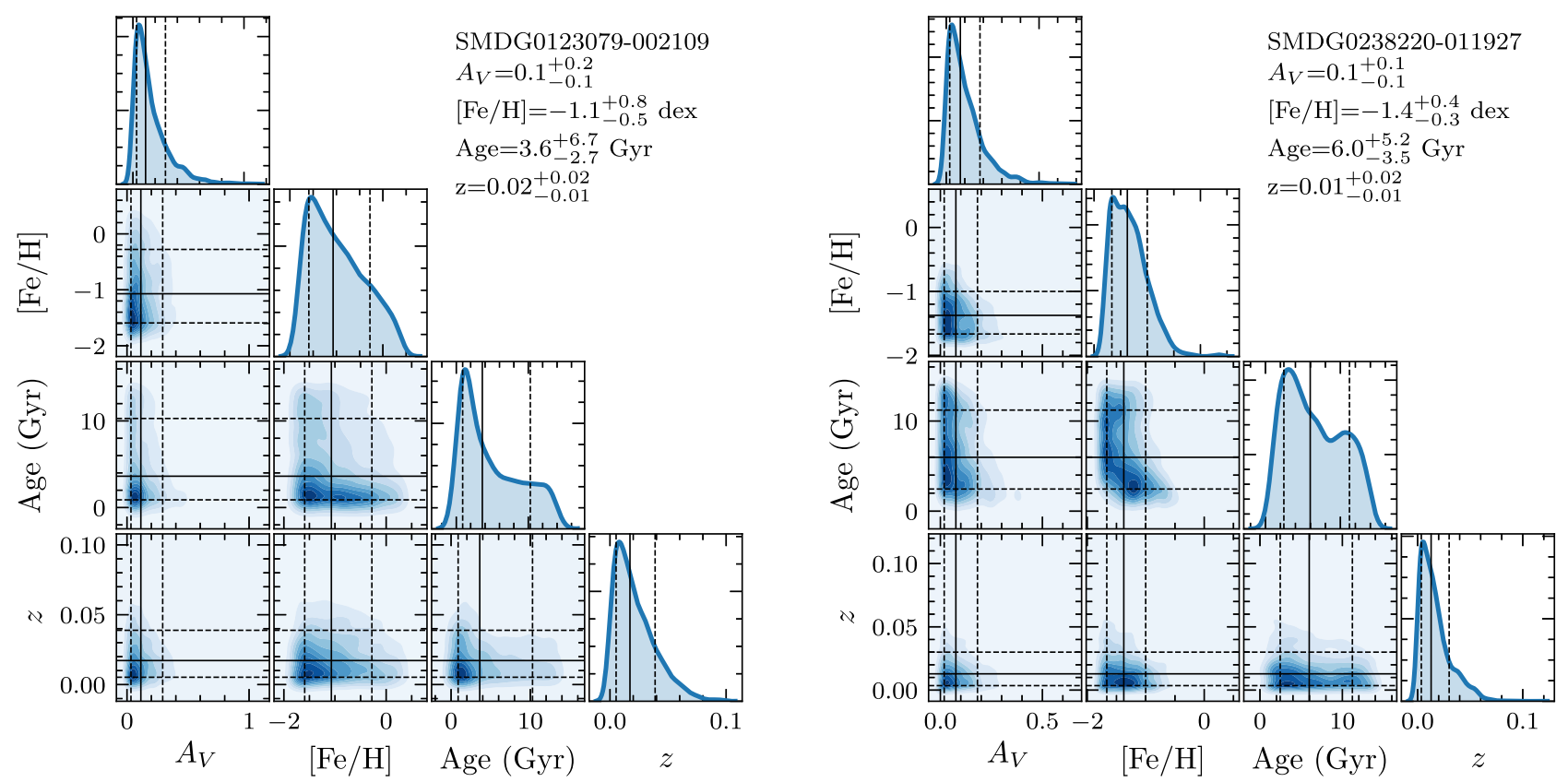

Figure 4. Sampled posterior distributions for two UDGs, SMDG0123079-002109 (left) and SMDG0238220-011927 (right), representing cases of the faint and bright end of the apparent magnitude distribution in our sample, respectively. The histograms along the diagonal contain the marginalized posterior distribution of parameters from where the resulting values and uncertainties are evaluated. The panels under the diagonal contain projections between pairs of variables, indicating how they are correlated. Solid lines mark the medians of the distributions, whereas dashed lines mark the $16 \%$ and $84 \%$ percentiles used to define the $1 \sigma$ uncertainties. A summary of the results is included in the upper right corner of each panel.

Table 1

Stellar Population Parameters for SMUDGes UDGs Obtained from SED Fitting of the S-PLUS Optical Data

\begin{tabular}{|c|c|c|c|c|c|}
\hline $\begin{array}{l}\text { Name } \\
\text { (1) }\end{array}$ & $\begin{array}{l}A_{V} \\
(2)\end{array}$ & $\begin{array}{c}{[\mathrm{Fe} / \mathrm{H}](\mathrm{dex})} \\
\text { (3) }\end{array}$ & $\begin{array}{c}\text { Age (Gyr) } \\
\text { (4) }\end{array}$ & $\begin{array}{c}z \\
(5)\end{array}$ & $\begin{array}{l}\log M_{\star} \\
\text { (6) }\end{array}$ \\
\hline SMD G0006543-000029 & $0.07_{-0.05}^{+0.12}$ & $-1.3_{-0.34}^{+0.7}$ & $2.8_{-2.1}^{+7.0}$ & $0.018_{-0.013}^{+0.020}$ & $7.5_{-1.1}^{+0.7}$ \\
\hline SMD G0016502-002756 & $0.11_{-0.08}^{+0.17}$ & $-1.0_{-0.5}^{+0.8}$ & $7.1_{-4.0}^{+4.5}$ & $0.021_{-0.015}^{+0.020}$ & $8.0_{-1.0}^{+0.6}$ \\
\hline SMD G0021031+004447 & $0.18_{-0.13}^{+0.28}$ & $-0.4_{-0.8}^{+0.6}$ & $8.0_{-4.5}^{+4.3}$ & $0.022_{-0.015}^{+0.021}$ & $7.9_{-0.9}^{+0.7}$ \\
\hline SMD G0025396+011515 & $0.10_{-0.07}^{+0.19}$ & $-1.1_{-0.5}^{+0.8}$ & $5.8_{-4.5}^{+5.5}$ & $0.019_{-0.013}^{+0.020}$ & $7.5_{-1.0}^{+0.7}$ \\
\hline SMD G0035569+010149 & $0.08_{-0.06}^{+0.14}$ & $-1.2_{-0.4}^{+0.6}$ & $3.1_{-2.3}^{+6.9}$ & $0.017_{-0.011}^{+0.021}$ & $7.4_{-1.0}^{+0.7}$ \\
\hline SMD G0045200-011839 & $0.07_{-0.05}^{+0.12}$ & $-1.3_{-0.4}^{+1.0}$ & $1.5_{-0.9}^{+6.0}$ & $0.018_{-0.013}^{+0.018}$ & $7.4_{-1.2}^{+0.7}$ \\
\hline SMD G0055526-011739 & $0.14_{-0.10}^{+0.24}$ & $-0.8_{-0.7}^{+0.8}$ & $7.4_{-4.6}^{+4.4}$ & $0.020_{-0.014}^{+0.022}$ & $7.5_{-1.1}^{+0.7}$ \\
\hline SMD G0058071-010201 & $0.09_{-0.07}^{+0.14}$ & $-1.2_{-0.4}^{+0.8}$ & $4.6_{-3.5}^{+6.1}$ & $0.021_{-0.015}^{+0.021}$ & $8.0_{-1.0}^{+0.6}$ \\
\hline SMD G0108359-002834 & $0.18_{-0.14}^{+0.28}$ & $-0.5_{-0.8}^{+0.6}$ & $8.0_{-4.4}^{+4.1}$ & $0.022_{-0.015}^{+0.024}$ & $7.9_{-1.1}^{+0.7}$ \\
\hline SMD G0113101-001223 & $0.10_{-0.07}^{+0.15}$ & $-1.1_{-0.5}^{+0.8}$ & $4.3_{-3.4}^{+6.6}$ & $0.020_{-0.014}^{+0.020}$ & $7.4_{-1.1}^{+0.7}$ \\
\hline
\end{tabular}

Note. Table sample containing only the first ten entries.

(This table is available in its entirety in machine-readable form.)

\subsection{Implications of the Estimated Redshifts to the Classification of UDG Candidates}

The most important implication of the redshift uncertainty is on the question of whether the UDG candidates are real UDGs, i.e., are they physically large, $R_{e} \geqslant 1.5 \mathrm{kpc}$. We showed in Section 4.4 that we are only able to minimally constrain photometric redshifts for the bright UDG candidates $\left(m_{r} \lesssim 19\right)$, and we rely on the prior distribution to estimate the redshifts of the fainter UDG candidates.

If, for the sake of argument, we consider the photometric redshift estimates to be correct, we can test whether the candidates can be classified as UDGs and whether this leads to any obvious irregularities. First, in Figure 6 we show the estimated effective radii of our UDG candidates as a function of the apparent magnitude, using the posterior distribution samples for the photometric redshift, and adopting the angular sizes, $R_{e}$, determined by SMUDGes. We use the apparent magnitude as the independent variable to emphasize that our ability to constrain the sizes is directly affected by the observed luminosity of the UDGs, but this does not reflect the actual size-luminosity relation that is expected to exist for UDGs if they are similar to other galaxies (e.g., Kormendy 1977). All but two candidates are larger than the UDG criterion with greater than $50 \%$ confidence. Of course, for the fainter systems this is principally a reflection of the adopted prior distribution, but for the brighter systems, where the determined redshift differs from the prior median, we have greater confidence that the physical sizes bear some resemblance to the truth. Second, the adopted redshifts do not lead to an unexpected set of very large $\left(R_{e}>6 \mathrm{kpc}\right)$ UDGs. As such, our determinations are not manifestly incorrect. 

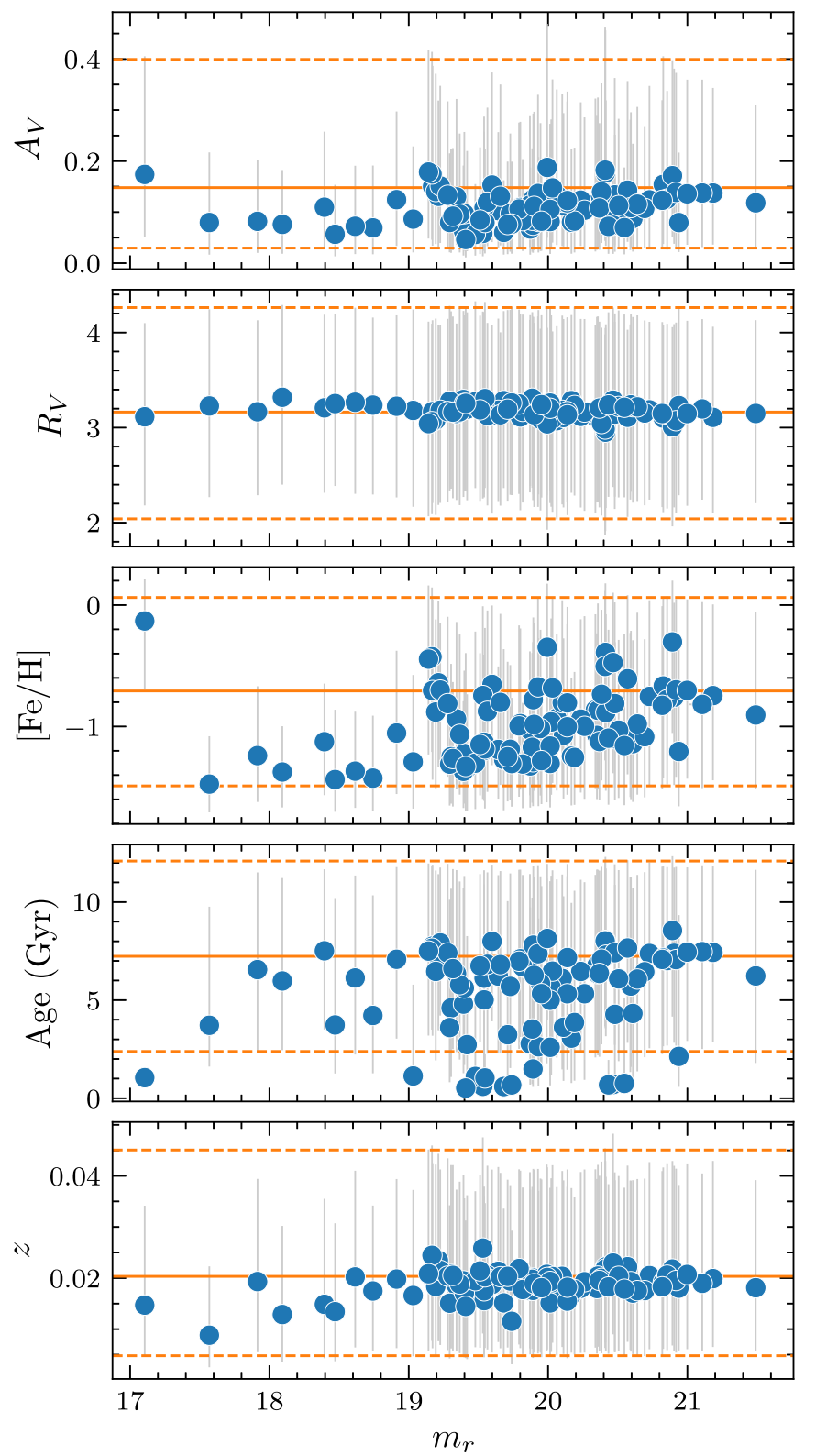

Figure 5. SED fitting parameters as a function of the $r$-band apparent magnitude. The blue circles indicate the posterior distribution median and the gray vertical lines represents the $1 \sigma$ uncertainties of the five free parameters, while the orange solid (dashed) lines indicate the median ( $\pm 1 \sigma$ uncertainties) of the prior distributions of the same parameters.

Given the limited redshift information contained in our observations, we are unable to conclude that our candidates are all real UDGs, but the bright ones are likely to be real UDGs, as well as the ones we discussed previously as confirmed through distance-by-association. For the sample as a whole, we argue based on volume considerations that they are likely to be farther away than our adopted median prior distance. The argument goes as follows. First, we specify that the maximum size of any UDG is $R_{e}=6 \mathrm{kpc}$, which sets a maximum distance for each of our candidates. The candidate can lie at any distance up to this maximum distance. Second, we assume that the parent population of our candidates is uniformly distributed throughout the local volume. Third, we claim that our selection is effectively independent of distance, within this volume, because it depends on surface brightness more than on luminosity. The latter

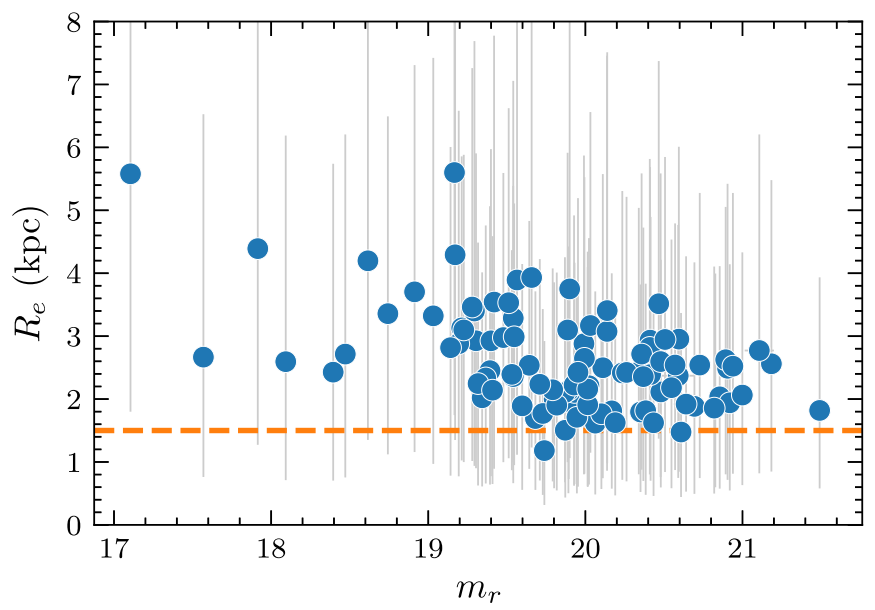

Figure 6. Effective radii of UDG candidates as a function of their apparent magnitude. The circles and their uncertainties indicate the median and the $1 \sigma$ uncertainties of the effective radius calculated using the posterior distributions of the SED fitting. The orange dashed line indicates the minimum physical radius of UDGs.

statement is not strictly correct, but is valid at the coarse level of this argument (Zaritsky et al. 2019). In such a scenario the mean distance to our candidates is $159 \pm 40 \mathrm{Mpc}$, or $z=0.036 \pm 0.01$, which is greater than our adopted median prior and supports the argument that the majority of candidates are indeed UDGs. In the next sections, we use the term UDG for all candidates, acknowledging that some of them might not meet the physical size criterion for UDGs.

\section{Discussion}

In this section we examine a variety of established galactic relations and properties, and place our UDG sample in context. We restrict our discussion to the stellar population properties and to only one variable that depends on the distance, the stellar mass, to avoid observed relations that may be contaminated by large correlations among parameters owing to our photometric redshift estimations.

\subsection{The Stellar Mass-Metallicity Relation of UDGs}

We begin this exploration by determining whether UDGs are similar to other LSB galaxies (see McConnachie 2012; Kirby et al. 2013), and thus follow the same stellar mass-metallicity relation as bright galaxies (Gallazzi et al. 2005). Previous studies found that UDGs usually conform to the stellar mass-metallicity relation defined by dwarf galaxies, but most of those UDGs are in or near clusters, such as Coma (Ferré-Mateu et al. 2018; Gu et al. 2018; Ruiz-Lara et al. 2018), with a few examples of UDGs not associated to clusters (Martínez-Delgado et al. 2016; Greco et al. 2018; Fensch et al. 2019).

In Figure 7 we show the stellar mass-metallicity relation and include our sample of UDGs. There is a large variety of data types, models, and methods involved in the determination of stellar populations of UDGs in the literature, and part of the scatter in the mass-metallicity and other relations may be attributed to that. For instance, Greco et al. (2018) have shown a difference of 0.3-0.5 dex in the metallicity of UDGs by simply changing from a single stellar population to an extended SFH. However, despite this important caveat, our measurements are consistent with previous work, with UDGs filling 


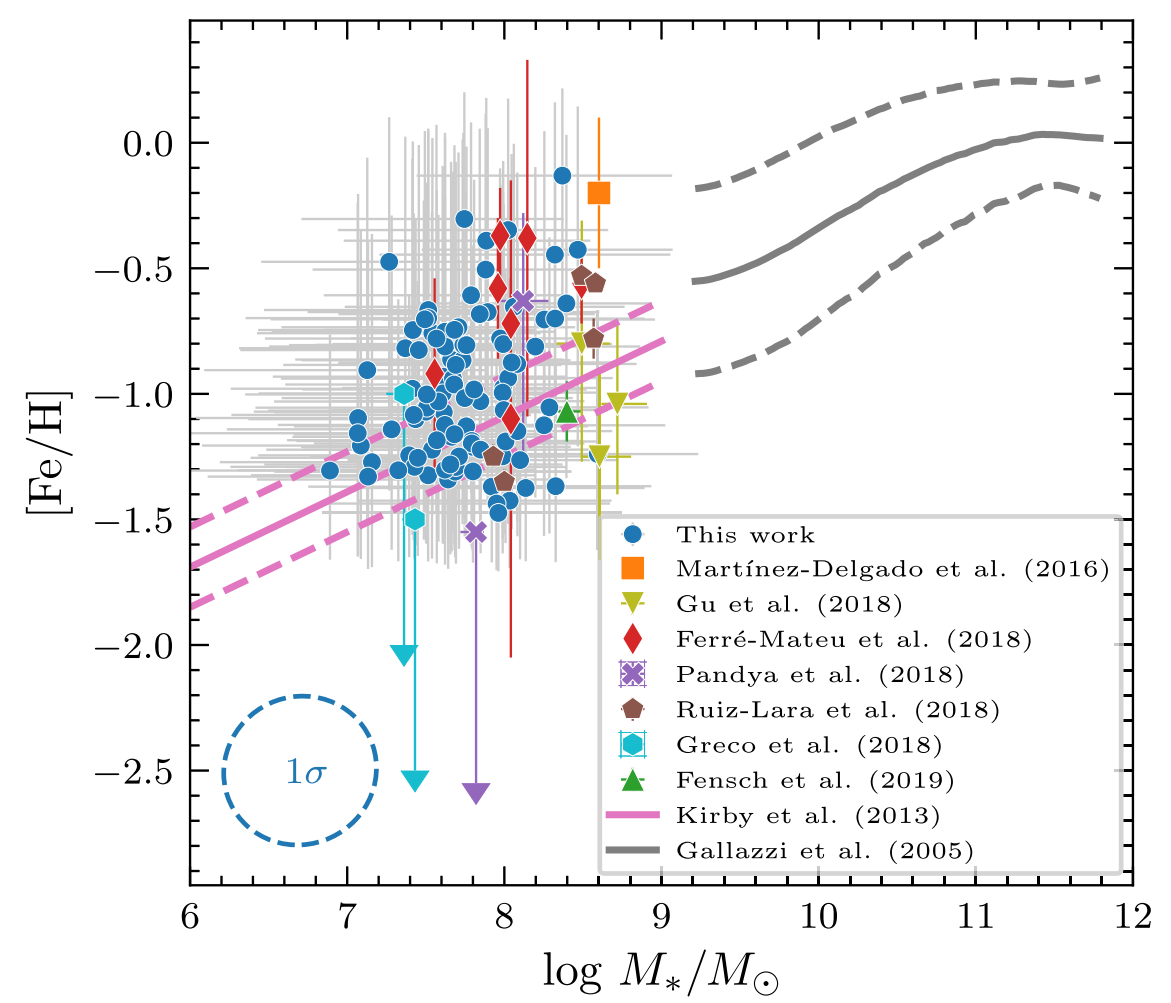

Figure 7. Stellar mass-metallicity relation of UDGs in our sample (filled blue circles) and from the literature (Martínez-Delgado et al. 2016; Ferré-Mateu et al. 2018; Greco et al. 2018; Gu et al. 2018; Pandya et al. 2018; Ruiz-Lara et al. 2018; Fensch et al. 2019). The pink solid and dashed lines are the mean and the scatter of the stellar mass-metallicity of dwarf galaxies around the Milky Way from Kirby et al. (2013). The gray lines are the mean and the standard deviation of the properties of bright galaxies from Gallazzi et al. (2005). The shape of the blue dashed ellipse in the bottom left indicates the mean $1 \sigma$ covariance between the parameters, where the direction of largest (smallest) variance corresponds to the major (minor) semiaxis.

part of the gap between dwarf and giant galaxies. In the bottom left of the figure, we include an ellipse whose shape shows the covariance between the two parameters, which indicates that the stellar mass and the metallicity are basically independent in our measurements.

Our large sample of galaxies allows for the observation of a range of metallicities that matches the variety of metallicities previously identified in the literature. However, the large uncertainties of and the censored limits on the range of metallicites do not allow for a reliable measure of the metallicity scatter for the UDGs in the sample. Overall, the location of the population of UDGs in the stellar massmetallicity diagram indicates a similarity with other dwarf LSB galaxies, such as those observed by Kirby et al. (2013). On average, the metallicities of the UDGs, as presented, are slightly larger than those predicted from the extrapolation of the relation derived from measurements of dwarf galaxies, but there are a few important considerations that favor the idea that the metallicity of UDGs follows the same relation as the dwarf galaxies.

First, the UDGs are not statistically far away from the dwarf sequence. Considering only our sample of UDGs, the mean difference between the measured metallicity and the expected metallicity from the Kirby et al. (2013) relation is 0.18 dex, which is similar to the scatter of the dwarf galaxies around the mean $(0.14 \mathrm{dex})$, and much smaller than the mean error in our measurements (0.6 dex). Second, there may be a systematic error in our measurements related to the assumed relation between the total metallicity and the iron abundance, $[\mathrm{M} / \mathrm{H}]=$ $[\mathrm{Fe} / \mathrm{H}]$, because the low metallicity stars used in the E-MILES models contain alpha elements. Ferré-Mateu et al. (2018) reported a few UDGs with significant overabundances of magnesium $(0 \lesssim[\mathrm{Mg} / \mathrm{Fe}] \lesssim 0.4$ for three out of four galaxies $)$ and Martín-Navarro et al. (2019) reported an even larger overabundance in DGSAT I, $[\mathrm{Mg} / \mathrm{Fe}]=1.5$. An average magnesium abundance of $[\mathrm{Mg} / \mathrm{Fe}] \approx 0.2 \mathrm{dex}$ is enough to account for the difference we find between the metallicity of our UDGs and that published for the dwarf galaxies. ${ }^{13}$ Finally, our SSP models are restricted to a lower limit of $[\mathrm{Fe} / \mathrm{H}]=-1.8$, and thus the metallicity of some UDGs in our sample may be overestimated. Note that a factor of two smaller distance, which would then render most of our candidates as nonUDGs, would lead to a factor of four lower stellar mass and would exacerbate the metallicity offset.

In conclusion, the metallicity of the UDGs is roughly consistent with that of other galaxies of similar stellar masses, the high-mass end of the dwarf sequence, and so do not show any signs of a different formation path than those galaxies.

\subsection{The Luminosity-weighted Ages of Field UDGs}

The reported ages of UDGs have usually been large, $>4 \mathrm{Gyr}$, but again almost all published results are for Coma galaxies (Ferré-Mateu et al. 2018; Gu et al. 2018; Ruiz-Lara et al. 2018). In the limited available examples of field UDGs, however, the reported luminosity-weighted ages have consistently been younger, with ages ranging from 1 to $3 \mathrm{Gyr}$ (Martínez-Delgado et al. 2016; Greco et al. 2018; Martín-Navarro et al. 2019). In fact, the UDG population in the field is expected to have a larger variety of colors than that of the clusters

\footnotetext{
${ }^{13}$ For the E-MILES models, $[\mathrm{Fe} / \mathrm{H}]=[\mathrm{M} / \mathrm{H}]-0.75[\mathrm{Mg} / \mathrm{Fe}]$.
} 


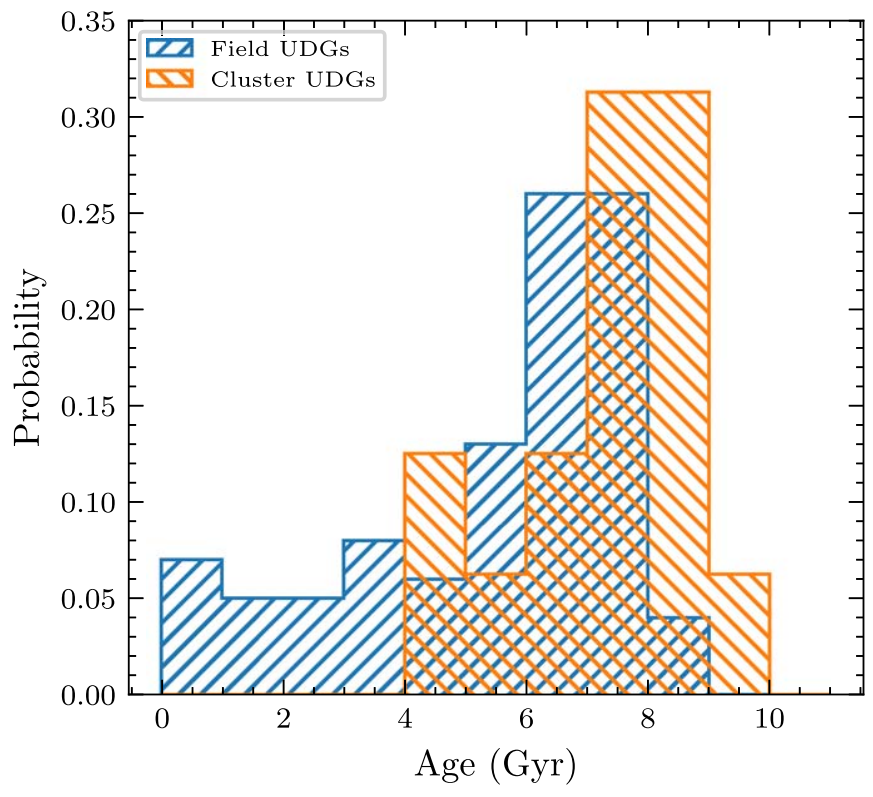

Figure 8. Comparison of the luminosity-weighted ages of our UDGs, which are primarily in the field, and those of UDGs in the literature (Ferré-Mateu et al. 2018; Gu et al. 2018; Pandya et al. 2018; Ruiz-Lara et al. 2018), which are primarily in clusters.

(Di Cintio et al. 2017) and there is observational support for this trend (van der Burg et al. 2016; Prole et al. 2019).

In Figure 8 we compare the luminosity-weighted age distribution of galaxies in our sample to that of UDGs observed in the Coma (Ferré-Mateu et al. 2018; Gu et al. 2018; RuizLara et al. 2018) and Virgo (Pandya et al. 2018) clusters, to contrast the distribution of ages in clusters and in the field. Both the field and cluster UDGs typically have intermediate ages, with a peak in the age histogram around $7 \mathrm{Gyr}$, but our sample also indicates a significant fraction of UDGs with ages smaller than 4 Gyr.

Considering that we only have luminosity-weighted ages, the results from the our analysis are expected to be biased toward the youngest populations within a galaxy. Therefore, a few different, nonexclusive scenarios can explain the additional fraction of UDGs with young ages. One possible explanation is the existence of different UDG formation channels not present in the cluster population (e.g., Liao et al. 2019). Other possibilities are that UDGs in the field might have more continuous star formation activity, presumably in the absence of cluster-related processes, such as harassment and ram pressure stripping, and that field UDGs have had a recent, even ongoing, star formation burst that outshines the older and more massive stellar component of the galaxy. Regardless of the detailed explanation, UDGs have been able to flourish in the field by forming stars until much more recently than UDGs in clusters.

\subsection{The Age-Metallicity Relation of UDGs}

We compare in Figure 9 the luminosity-weighted agemetallicity relation for UDGs, both in the field and in galaxy clusters, to that of bright galaxies. Within our own field UDG sample, there appears to be a correlation between age and metallicity, but considering the existence of a well-known agedegeneracy problem, we first inspect whether this is causing the observed relation.
The original age-deneracy problem (Worthey 1994) indicates that the colors of an old population are similar to those of another population three times older and with half the metallicity. This degeneracy is specific for broad bands and old stellar systems, and thus it is unclear whether this holds in our analysis. However, it is an important cautionary note to any stellar population analysis, as degeneracies are bound to happen in SED fitting. As we indicate with the error ellipse in the bottom of the figure, there is a correlation between the age and the metallicity in our posterior distributions that is similar to the known age-metallicity degeneracy. However, the observed relation between the ages and metallicities of our UDGs does not have a slope in the same direction as the agedegeneracy relation. Therefore, we conclude that the observed relation between ages and metallicities in our UDG sample is not driven by the age-metallicity degeneracy, and thus we are able to discuss some properties of the observed relation.

For the old UDGs (age $\gtrsim 6$ Gyr), the age-metallicity relation follows a similar pattern to that of bright galaxies (Gallazzi et al. 2005), in the sense that younger systems have low metallicity and older systems have high metallicity, although with different slope and offset. The old UDGs have metallicities similar to those reported in other works for UDGs in clusters, but this possible age-metallicity relation was not hinted at in previous work.

The young UDGs have a flat age-metallicity relation, but the modeling limitation in the range of very low metallicities limits us in reaching further conclusions as to whether the flattening in the relation is real or a result of the modeling restriction. The location of our young UDGs in this space is similar to that of the field UDGs from Greco et al. (2018), which were suggested to be currently star forming.

Interestingly, there are also a few young UDGs $(t \lesssim 1 \mathrm{Gyr})$ with relatively high metallicities $([\mathrm{Fe} / \mathrm{H}] \approx-0.5 \mathrm{dex})$, populating the location of more massive galaxies. These UDGs are located in the age-metallicity plane in a location similar to that of DGSAT I, a passive, field UDG found in the Pisces-Perseus supercluster filament (Martínez-Delgado et al. 2016). A visual inspection of our young, metal-rich UDGs does not suggest current tidal interactions with bright galaxies, and thus it is not likely that these particular UDGs have tidal origins, which could have explained their higher metallicity. A more likely scenario is that these cases indicate more massive UDGs that have had a recent burst in star formation, such that their luminosity-weighted metallicities are driven by a potentially old, mass-dominant stellar component, while their luminosityweighted ages are driven by a less massive, young component.

\subsection{The Stellar Mass-Age Relation}

In Figure 10 we present the relation between the stellar mass and the luminosity-weighted age for our UDG sample and more massive galaxies. Similar to the age-metallicity relation, we also observe a correlation between the ages and the stellar masses. However, in this case, the error ellipse in the bottom of the figure indicates that the observed correlation may be caused by a degeneracy in the parameters, and thus we do not have any confidence that this relation actually exists.

Most old UDGs (age $\gtrsim 6$ Gyr) follow the stellar massmass-weighted age relation observed by Thomas et al. (2005), extrapolated to the UDG regime. We explain this agreement by noting that the luminosity-weighted and mass-weighted ages converge the longer a galaxy is not forming stars. However, the 


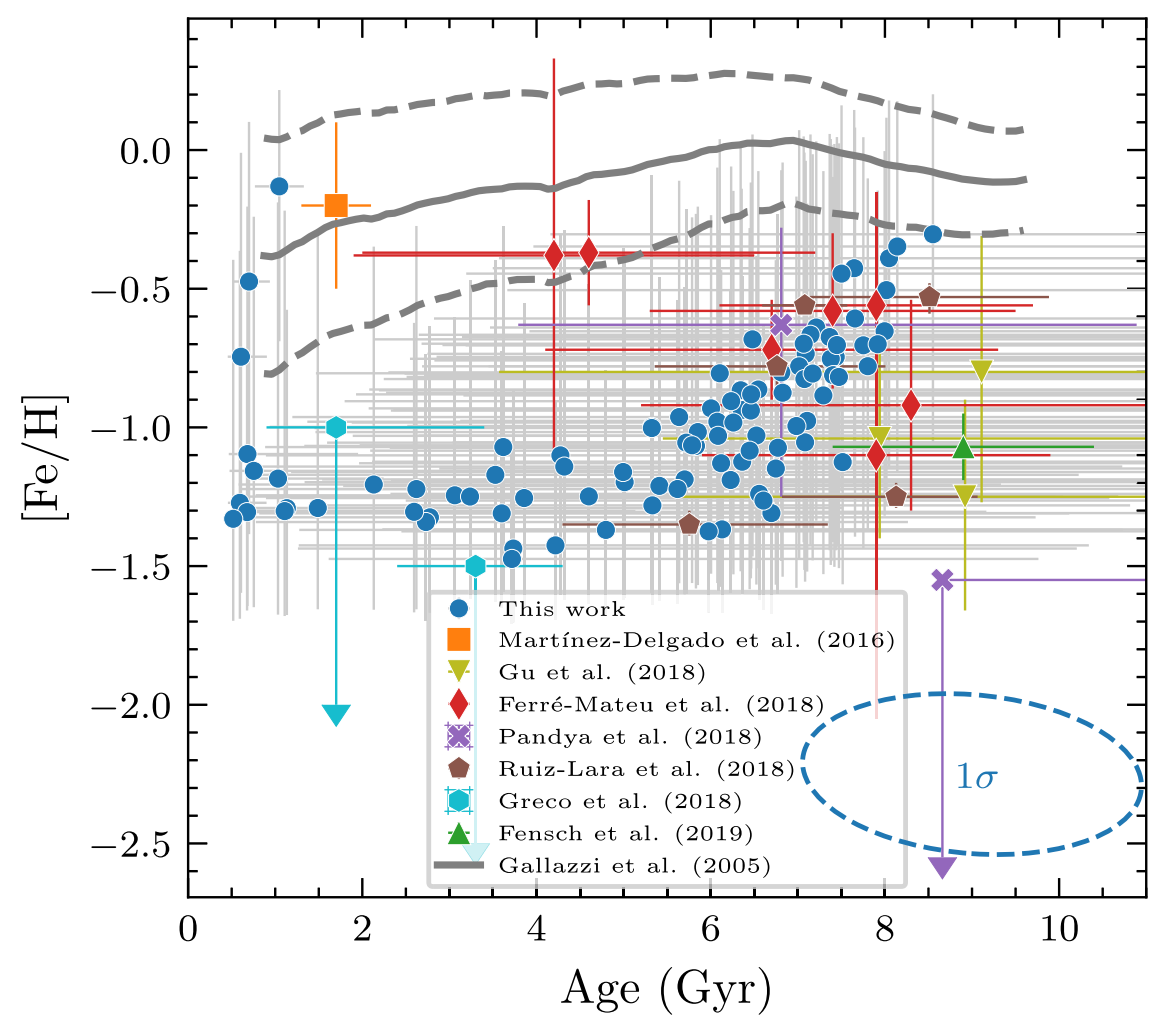

Figure 9. Comparison between luminosity-weighted ages and metallicities of UDGs in the field (this work) and from the literature (Martínez-Delgado et al. 2016; Ferré-Mateu et al. 2018; Greco et al. 2018; Gu et al. 2018; Pandya et al. 2018; Ruiz-Lara et al. 2018; Fensch et al. 2019). Solid and dashed lines are the mean and the standard deviation of the relation for bright galaxies (Gallazzi et al. 2005). The blue dashed ellipse in the bottom right indicates the mean $1 \sigma$ covariance between the parameters.

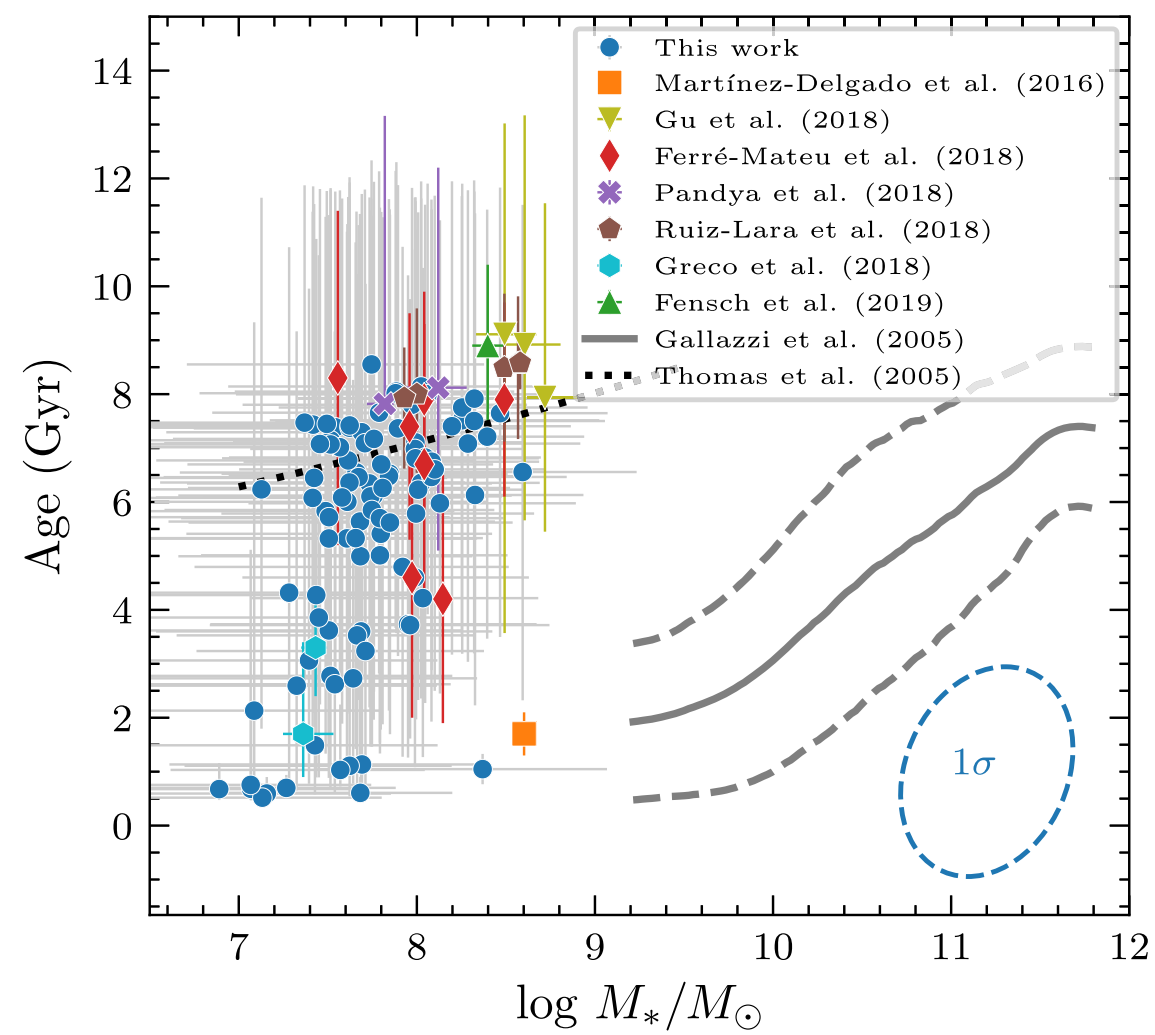

Figure 10. Same as Figure 9 for the relation between stellar mass and luminosity-weighted age for UDGs and bright galaxies. 
young UDGs are displaced from the relation of Thomas et al. (2005), and have ages similar to those in the low-mass end of the Gallazzi et al. (2005) relation. We expect these young field UDGs to move upward in this diagram when they eventually stop forming stars.

\section{Summary and Conclusion}

UDGs are large, low surface brightness galaxies. Although such systems are now known in significant numbers, establishing physical characteristics has proven to be challenging even when using the largest telescopes of this generation. Field UDGs, in particular, have barely been studied. In this work, we perform the first systematic study of the stellar populations of field UDGs combining the deep and large area search of UDGs performed by the SMUDGes survey (Zaritsky et al. 2019) with the multiband capabilities of the S-PLUS survey (Mendes de Oliveira et al. 2019). Covering an area of $330 \mathrm{deg}^{2}$ in the Stripe 82 region, we fit SEDs to a sample of 100 field UDGs, representing the largest sample of UDGs (field or cluster) for which ages and metallicities have been measured to date.

We constrain our Bayesian SED fitting method with up to 12 broad and narrow bands from S-PLUS, resulting in estimated luminosity-weighted ages, metallicities, and stellar masses of the field UDGs. While stellar masses and metallicities are mostly in agreement with previous studies, we observe a number of UDGs with ages younger than those found in cluster. This result suggests that UDGs in the field may have extended star formation histories that may, in some cases, extend to the current time, contrasting with the typical old ages of UDGs found in clusters. We also find a few cases of relatively high-metallicity UDGs $([\mathrm{Fe} / \mathrm{H}] \approx-0.5)$ with young ages (ages $\lesssim 1$ Gyr) without clear indications of tidal interactions, which suggest ongoing episodes of star formation among the most massive UDGs.

Previous studies have already indicated that UDGs may represent the extension of normal galaxy properties rather than arise from a disconnected, new path of galaxy formation, but these conclusions have been based on small samples of galaxies (Beasley \& Trujillo 2016; Zaritsky 2017) or models (Amorisco \& Loeb 2016). By placing a large sample of field UDGs in stellar population scaling relations, we are able to confirm that UDGs, as a population, are similar to dwarf and giant galaxies. There are still puzzles to solve, such as the large globular cluster abundances in the largest UDGs (van Dokkum et al. 2017; Toloba et al. 2018) and the offset from the baryonic Tully-Fisher relation (Mancera Piña et al. 2019), but we conclude that these should arise naturally from considering a broader range of galaxies within the current picture of galaxy formation (Martin et al. 2019) rather than any exotic processes (Bennet et al. 2018). Of course, these statements apply to the general case and individual exceptions, where UDGs form in tidal tails, for example, are not excluded.

Despite the improvement in sample size in this work, there is still much to be gained from even larger samples. In particular, we want to apply the same analysis methods to UDGs in a range of environments, including massive clusters, to more confidently compare results. Even larger samples will enable us to test for further dependencies on UDG mass, environment, and morphology. Both SMUDGes and S-PLUS are still in their early phases. A much larger area of the sky will be probed by both surveys in the next years, increasing the sample for which this type of analysis can be replicated into the thousands.
The authors acknowledge the anonymous referee for his/her comments. We are thankful to Stavros Akras, Yoli Jiménez Teja, Marco Grossi, Alvaro Alvarez-Candal, José Luis Nilo Castellón, Paulo Lopes, Kanak Saha, Eduardo Telles, and Ana Chies Santos for providing comments and suggestions. C.E.B. and C.M.dO. gratefully acknowledge the São Paulo Research Foundation (FAPESP), grants 2009/54202-8, 2016/17119-9, 2019/11910-4, 2011/51680-6, 2016/12331-0, and 2018/24389-8. D.Z., R.D., and H.Z. gratefully acknowledge financial support from NSF AST-1713841. PC acknowledges support from FAPESP project 2018/05392-8, and Conselho Nacional de Desenvolvimento Científico e Tecnológico (CNPq) project 310041/2018-0. L.S. thanks the FAPESP scholarship grant 2016/21664-2. F.R.H. thanks FAPESP for the financial support of grants 2019/23141-5 and 2018/21661-9. J.A.H.J. thanks the Brazilian institution CNPq for financial support through a postdoctoral fellowship (project 150237/2017-0) and the Chilean institution CONICYT, Programa de Astronomía, Fondo ALMA-CONICYT 2017, Código de proyecto 31170038. The T80South robotic telescope (Mendes de Oliveira et al. 2019) was founded as a partnership between Universidade de São Paulo, the Observatório Nacional (ON), the Universidade Federal de Sergipe (UFS), and the Federal University of Santa Catarina (UFSC), with important financial and practical contributions from other collaborating institutes in Brazil, Chile (Universidad de La Serena), and Spain (CEFCA). This work has made use of the computing facilities of the Laboratory of Astroinformatics (Instituto de Astronomia, Geofísica e Ciências Atmosféricas, Departamento de Astronomia/ USP, NAT/Unicsul), whose purchase was made possible by FAPESP (grant 2009/54006-4) and the INCT-A. This research has made use of the NASA/IPAC Extragalactic Database (NED), which is operated by the Jet Propulsion Laboratory, California Institute of Technology, under contract with the National Aeronautics and Space Administration. We warmly thank Mariano Moles, Javier Cenarro, Tamara Civera, Sergio Chueca, Javier Hernandez Fuertes, Alessandro Ederoclite, David Cristobal, Antonio Marín Franch, Jesus Varella, and Hector Vazquez Ramio - the success of the S-PLUS project relies on the dedication of these and other CEFCA staff members in building OAJ and running J-PLUS and J-PAS.

Facility: S-PLUS.

Software: astropy (Astropy Collaboration et al. 2013), matplotlib (Hunter 2007), numpy (van der Walt et al. 2011), pymc3 (Salvatier et al. 2016), scipy (Jones et al. 2001), photutils (Bradley et al. 2019).

\section{ORCID iDs}

C. E. Barbosa (1D https://orcid.org/0000-0002-5292-2782

D. Zaritsky (1) https://orcid.org/0000-0002-5177-727X

R. Donnerstein (iD https://orcid.org/0000-0001-7618-8212

H. Zhang (i) https://orcid.org/0000-0002-0123-9246

A. Dey (iD https://orcid.org/0000-0002-4928-4003

C. Mendes de Oliveira (i) https://orcid.org/0000-00025267-9065

P. Coelho (1D https:// orcid.org/0000-0003-1846-4826

A. Cortesi (i) https://orcid.org/0000-0002-0620-136X

F. R. Herpich (iD https://orcid.org/0000-0001-7907-7884

E. Pereira (i) https://orcid.org/0000-0002-1564-2933

A. Werle (iD https://orcid.org/0000-0002-4382-8081

R. A. Overzier (i) https://orcid.org/0000-0002-8214-7617

R. Cid Fernandes (iD https://orcid.org/0000-0001-9672-0296

W. Schoenell (iD https://orcid.org/0000-0002-4064-7234 


\section{References}

Abraham, R. G., \& van Dokkum, P. G. 2014, PASP, 126, 55

Allen, C. W. 1976, Astrophysical Quantities (3rd ed.; London: Athlone)

Amorisco, N. C., \& Loeb, A. 2016, MNRAS, 459, L51

Amorisco, N. C., Monachesi, A., Agnello, A., \& White, S. D. M. 2018, MNRAS, 475, 4235

Astropy Collaboration, Robitaille, T. P., Tollerud, E. J., et al. 2013, A\&A, 558, A33

Beasley, M. A., Romanowsky, A. J., Pota, V., et al. 2016, ApJL, 819, L20

Beasley, M. A., \& Trujillo, I. 2016, ApJ, 830, 23

Benitez, N., Dupke, R., Moles, M., et al. 2014, arXiv:1403.5237

Bennet, P., Sand, D. J., Zaritsky, D., et al. 2018, ApJL, 866, L11

Bouchet, P., Lequeux, J., Maurice, E., Prevot, L., \& Prevot-Burnichon, M. L. 1985, A\&A, 149, 330

Bradley, L., Sipőcz, B., Robitaille, T., et al. 2019, astropy/photutils: v0.6, Zenodo, doi: $10.5281 /$ zenodo. 2533376

Byler, N., Dalcanton, J. J., Conroy, C., \& Johnson, B. D. 2017, ApJ, 840, 44 Calzetti, D., Armus, L., Bohlin, R. C., et al. 2000, ApJ, 533, 682

Cardelli, J. A., Clayton, G. C., \& Mathis, J. S. 1989, ApJ, 345, 245

Carleton, T., Errani, R., Cooper, M., et al. 2019, MNRAS, 485, 382

Cenarro, A. J., Moles, M., Cristóbal-Hornillos, D., et al. 2019, A\&A, 622, A176

Chabrier, G. 2003, PASP, 115, 763

Chan, T. K., Kereš, D., Wetzel, A., et al. 2018, MNRAS, 478, 906

Conselice, C. J. 2018, RNAAS, 2, 43

Dalcanton, J. J., Spergel, D. N., Gunn, J. E., Schmidt, M., \& Schneider, D. P. 1997, AJ, 114, 635

DESI Collaboration, Aghamousa, A., Aguilar, J., et al. 2016a, arXiv:1611.00036

DESI Collaboration, Aghamousa, A., Aguilar, J., et al. 2016b, arXiv:1611.00037

Dey, A., Schlegel, D. J., Lang, D., et al. 2019, AJ, 157, 168

Di Cintio, A., Brook, C. B., Dutton, A. A., et al. 2017, MNRAS, 466, L1

Disney, M. J. 1976, Natur, 263, 573

Driver, S. P., Hill, D. T., Kelvin, L. S., et al. 2011, MNRAS, 413, 971

Driver, S. P., Norberg, P., Baldry, I. K., et al. 2009, A\&G, 50, 5.12

Fensch, J., van der Burg, R. F. J., Jeřábková, T., et al. 2019, A\&A, 625, A77

Ferré-Mateu, A., Alabi, A., Forbes, D. A., et al. 2018, MNRAS, 479, 4891

Fioc, M., \& Rocca-Volmerange, B. 1999, arXiv:astro-ph/9912179

Fitzpatrick, E. L., \& Massa, D. 1986, ApJ, 307, 286

Foreman-Mackey, D., Hogg, D. W., Lang, D., \& Goodman, J. 2013, PASP, 125,306

Gallazzi, A., Charlot, S., Brinchmann, J., White, S. D. M., \& Tremonti, C. A. 2005, MNRAS, 362, 41

Gelfand, A. E., \& Smith, A. F. M. 1990, J. Am. Stat. Assoc., 85, 398

Gelman, A., Carlin, J. B., Stern, H. S., \& Rubin, D. B. 2004, Bayesian Data Analysis (2nd ed.; London: Chapman)

Gonzalez, A. H., Zaritsky, D., Dalcanton, J. J., \& Nelson, A. 2001, ApJS, 137, 117

González, N. M., Smith Castelli, A. V., Faifer, F. R., Escudero, C. G., \& Cellone, S. A. 2018, A\&A, 620, A166

Graham, A. W., \& Driver, S. P. 2005, PASA, 22, 118

Greco, J. P., Goulding, A. D., Greene, J. E., et al. 2018, ApJ, 866, 112

Gu, M., Conroy, C., Law, D., et al. 2018, ApJ, 859, 37

Hastings, W. K. 1970, Biometrika, 57, 97

Hoffman, M. D., \& Gelman, A. 2011, arXiv:1111.4246

Hunter, J. D. 2007, CSE, 9, 90

Impey, C., Bothun, G., \& Malin, D. 1988, ApJ, 330, 634

Jiang, F., Dekel, A., Freundlich, J., et al. 2019, MNRAS, 487, 5272

Jones, E., Oliphant, T., Peterson, P., et al. 2001, SciPy: Open Source Scientific Tools for Python, http://www.scipy.org/

Kadowaki, J., Zaritsky, D., \& Donnerstein, R. L. 2017, ApJL, 838, L21

Kirby, E. N., Cohen, J. G., Guhathakurta, P., et al. 2013, ApJ, 779, 102

Kormendy, J. 1977, ApJ, 218, 333

Leisman, L., Haynes, M. P., Janowiecki, S., et al. 2017, ApJ, 842, 133

Leitherer, C., Schaerer, D., Goldader, J. D., et al. 1999, ApJS, 123, 3

Liao, S., Gao, L., Frenk, C. S., et al. 2019, MNRAS, 490, 5182

Makarov, D. I., Sharina, M. E., Karachentseva, V. E., \& Karachentsev, I. D. 2015, A\&A, 581, A82

Mancera Piña, P. E., Fraternali, F., Adams, E. A. K., et al. 2019, ApJL, 883, L33

Marín-Franch, A., Chueca, S., Moles, M., et al. 2012, Proc. SPIE, 8450 , $84503 \mathrm{~S}$

Martin, G., Kaviraj, S., Laigle, C., et al. 2019, MNRAS, 485, 796

Martínez-Delgado, D., Läsker, R., Sharina, M., et al. 2016, AJ, 151, 96

Martín-Navarro, I., Romanowsky, A. J., Brodie, J. P., et al. 2019, MNRAS, 484,3425
McConnachie, A. W. 2012, AJ, 144, 4

Mendes de Oliveira, C., Ribeiro, T., Schoenell, W., et al. 2019, MNRAS, 489,241

Mihos, J. C., Durrell, P. R., Ferrarese, L., et al. 2015, ApJL, 809, L21

Molino, A., Costa-Duarte, M. V., Sampedro, L., et al. 2019, arXiv:1907.06315 Ogiya, G. 2018, MNRAS, 480, L106

Oke, J. B. 1964, ApJ, 140, 689

Oke, J. B., \& Gunn, J. E. 1983, ApJ, 266, 713

Pandya, V., Romanowsky, A. J., Laine, S., et al. 2018, ApJ, 858, 29

Peng, C. Y., Ho, L. C., Impey, C. D., \& Rix, H.-W. 2002, AJ, 124, 266

Peng, C. Y., Ho, L. C., Impey, C. D., \& Rix, H.-W. 2010, AJ, 139, 2097

Peng, E. W., \& Lim, S. 2016, ApJL, 822, L31

Prevot, M. L., Lequeux, J., Maurice, E., Prevot, L., \& Rocca-Volmerange, B. 1984, A\&A, 132, 389

Prole, D. J., van der Burg, R. F. J., Hilker, M., \& Davies, J. I. 2019, MNRAS, 488, 2143

Ricciardelli, E., Vazdekis, A., Cenarro, A. J., \& Falcón-Barroso, J. 2012, MNRAS, 424, 172

Román, J., Beasley, M. A., Ruiz-Lara, T., \& Valls-Gabaud, D. 2019, MNRAS, 486,823

Román, J., \& Trujillo, I. 2017, MNRAS, 468, 4039

Rong, Y., Guo, Q., Gao, L., et al. 2017, MNRAS, 470, 4231

Ruiz-Lara, T., Beasley, M. A., Falcón-Barroso, J., et al. 2018, MNRAS, 478, 2034

Safarzadeh, M., \& Scannapieco, E. 2017, ApJ, 850, 99

Salvatier, J., Wiecki, T. V., \& Fonnesbeck, C. 2016, PeerJ Computer Science, 2, e55

Sandage, A., \& Binggeli, B. 1984, AJ, 89, 919

Savage, B. D., \& Mathis, J. S. 1979, ARA\&A, 17, 73

Schlafly, E. F., \& Finkbeiner, D. P. 2011, ApJ, 737, 103

Schlegel, D., Abdalla, F., Abraham, T., et al. 2011, arXiv:1106.1706

Schlegel, D. J., Finkbeiner, D. P., \& Davis, M. 1998, ApJ, 500, 525

Schombert, J. M., \& Bothun, G. D. 1988, AJ, 95, 1389

Schwartzenberg, J. M., Phillipps, S., Smith, R. M., Couch, W. J., \& Boyle, B. J. 1995, MNRAS, 275, 121

Serra, P., \& Trager, S. C. 2007, MNRAS, 374, 769

Shi, D. D., Zheng, X. Z., Zhao, H. B., et al. 2017, ApJ, 846, 26

Sifón, C., van der Burg, R. F. J., Hoekstra, H., Muzzin, A., \& Herbonnet, R. 2018, MNRAS, 473, 3747

Smith Castelli, A. V., Faifer, F. R., \& Escudero, C. G. 2016, A\&A, 596, A23 Sprayberry, D., Impey, C. D., Irwin, M. J., \& Bothun, G. D. 1997, ApJ, 482, 104

Stecher, T. P. 1965, ApJ, 142, 1683

Taylor, E. N., Hopkins, A. M., Baldry, I. K., et al. 2011, MNRAS, 418, 1587

Thomas, D., Maraston, C., Bender, R., \& Mendes de Oliveira, C. 2005, ApJ, 621,673

Toloba, E., Lim, S., Peng, E., et al. 2018, ApJL, 856, L31

van der Burg, R. F. J., Hoekstra, H., Muzzin, A., et al. 2017, A\&A, 607, A79

van der Burg, R. F. J., Muzzin, A., \& Hoekstra, H. 2016, A\&A, 590, A20

van der Walt, S., Colbert, S. C., \& Varoquaux, G. 2011, CSE, 13, 22

van Dokkum, P., Abraham, R., Romanowsky, A. J., et al. 2017, ApJL, 844, L11

van Dokkum, P., Cohen, Y., Danieli, S., et al. 2018a, ApJL, 856, L30

van Dokkum, P., Danieli, S., Cohen, Y., et al. 2018b, Natur, 555, 629

van Dokkum, P., Wasserman, A., Danieli, S., et al. 2019, ApJ, 880, 91

van Dokkum, P. G., Abraham, R., Merritt, A., et al. 2015, ApJL, 798, L45

Vanhatalo, J., Jylanki, P., \& Vehtari, A. 2009, in Proc. Advances in Neural Information Processing Systems 22 Conf., ed. Y. Bengio et al. (Red Hook, NY: Curran Associates), 1910, http://papers.nips.cc/paper/3806-gaussianprocess-regression-with-student-t-likelihood.pdf

Vazdekis, A., Casuso, E., Peletier, R. F., \& Beckman, J. E. 1996, ApJS, 106,307

Vazdekis, A., Koleva, M., Ricciardelli, E., Röck, B., \& Falcón-Barroso, J. 2016, MNRAS, 463, 3409

Vazdekis, A., Sánchez-Blázquez, P., Falcón-Barroso, J., et al. 2010, MNRAS, 404, 1639

Venhola, A., Peletier, R., Laurikainen, E., et al. 2017, A\&A, 608, A142

Werle, A., Cid Fernandes, R., Vale Asari, N., et al. 2019, MNRAS, 483, 2382

Willmer, C. N. A. 2018, ApJS, 236, 47

Worthey, G. 1994, ApJS, 95, 107

Yagi, M., Koda, J., Komiyama, Y., \& Yamanoi, H. 2016, ApJS, 225, 11

Yozin, C., \& Bekki, K. 2015, MNRAS, 452, 937

Zaritsky, D. 2017, MNRAS, 464, L110

Zaritsky, D., Donnerstein, R., Dey, A., et al. 2019, ApJS, 240, 1 